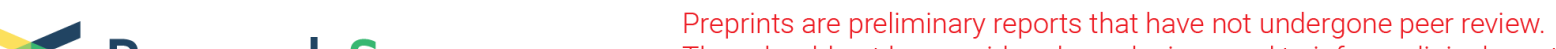 Research Square
Thr ey should not be considered conclusive, used to inform clinical practice,
of the media as validated information.
}

\section{A diencephalic circuit for opioid analgesia but not positive reinforcement}

\author{
Maggie Waung \\ University of California, San Francisco \\ Kayla Maanum \\ University of California, San Francisco \\ Joseph Driscoll \\ University of California, San Francisco \\ Chris O'Brien \\ Rutgers University \\ Svetlana Bryant \\ Rutgers University \\ Kasra Mansourian \\ University of California, San Francisco \\ Marisela Morales \\ National Institute on Drug Abuse https://orcid.org/0000-0002-3845-9402 \\ David Barker \\ Rutgers University \\ Elyssa Margolis ( $\sim$ Elyssa.Margolis@ucsf.edu ) \\ University of California, San Francisco https://orcid.org/0000-0001-8777-302X
}

\section{Article}

Keywords: Mu opioid receptor agonists, epithalamic lateral habenula, opioid analgesia

Posted Date: January 6th, 2021

DOl: https://doi.org/10.21203/rs.3.rs-125555/v1

License: (c) (i) This work is licensed under a Creative Commons Attribution 4.0 International License.

Read Full License

Version of Record: A version of this preprint was published at Nature Communications on February 9th, 2022. See the published version at https://doi.org/10.1038/s41467-022-28332-6. 
3 Maggie W. Waung ${ }^{1}$, Kayla A. Maanum ${ }^{1}$, Joseph R. Driscoll ${ }^{1}$, Chris O’Brien ${ }^{2}$, Svetlana Bryant ${ }^{2}$, Kasra

4 Mansourian $^{1}$, Marisela Morales ${ }^{3}$, David J. Barker ${ }^{2,3}$, and Elyssa B. Margolis ${ }^{1 * \dagger}$

6 'UCSF Weill Institute for Neuroscience, Department of Neurology, University of California,

7 San Francisco, CA, United States

$8 \quad{ }^{2}$ Department of Psychology, Rutgers University, New Brunswick, NJ, United States

$9{ }^{3}$ National Institute on Drug Abuse, Neuronal Networks Section, National Institutes of Health, Baltimore, 10 MD, United States

11

$12 *$ Corresponding Author

13 †Email: Elyssa.Margolis@ucsf.edu

14

15 Title- 10; Abstract- 149, Introduction- 316, Results- 2574, Discussion- 1326; Figures 5, 8 extended data 16 figures; Tables- 1 Supplementary Table

17 18 19 20 
Mu opioid receptor (MOR) agonists are the most effective analgesics, but their use risks respiratory depression and addiction. The epithalamic lateral habenula $(\mathrm{LHb})$ is a critical site that signals aversive states, often via indirect inhibition of reward circuitry, and MORs are highly expressed in the LHb. We found that the $\mathrm{LHb}$ is a potent site for both MOR-agonist analgesia. Strikingly, LHb MOR activation generates negative reinforcement but is not rewarding in the absence of noxious input. While the LHb receives inputs from multiple sites, we found that inputs from the lateral preoptic area of the hypothalamus (LPO) are excited by noxious stimulation, express MOR mRNA, and are preferentially targeted by MOR selective agonists. Critically, optogenetic stimulation of LHb-projecting LPO neurons produces an aversive state relieved by LHb MOR activation. Therefore targeting this MOR sensitive forebrain circuit can relieve pain yet lower the risk of misuse by pain free individuals.

\section{Introduction}

34 Opioids are the most effective pain medications, but the risk of overdose and opioid use disorder limits their clinical utility. Uncoupling the analgesic actions of opioids from those that underlie positive reinforcement is a longstanding goal for pharmacotherapeutic development. Identifying circuits that can drive relief of ongoing pain but not reward in the absence of pain is a critical step towards this goal. The lateral habenula $(\mathrm{LHb})$ may participate in such a circuit, which is not only activated in a pain setting ${ }^{1-5}$ but also by other aversive states including reward omission ${ }^{6}$, and animal models of depression ${ }^{7}$. Several CNS sites involved in pain signaling with reported strong inputs to the LHb include the lateral hypothalamus $(\mathrm{LH})^{8,9}$ and anterior cingulate cortex $(\mathrm{ACC})^{10-12}$. Furthermore, efferents from the LHb target painresponsive regions including the lateral periaqueductal gray, dorsal raphe, and parabrachial nucleus ${ }^{13,14}$.

43 While morphine injections that covered a combination of the $\mathrm{LHb}$, medial habenula, and posteromedial 
44 thalamus reduce pain-related behavior in an acute pain model ${ }^{15}$, whether these effects are due to MOR

45 activation specifically in the $\mathrm{LHb}$ is an open question.

46 Since increased activity in LHb neurons encodes aversive states including ongoing pain, inhibition of this

47 activity should relieve pain and generate negative reinforcement. We previously found that MOR

48 activation can decrease neural activity in the $\mathrm{LHb}$ via both postsynaptic hyperpolarization and inhibition

49 of glutamate release onto subsets of $\mathrm{LHb}$ neurons in naïve animals ${ }^{16}$. Here we investigated the specific

50 LHb input circuit and synaptic mechanism by which MOR activation in the LHb produces pain relief.

51 Among six potential inputs to the $\mathrm{LHb}$, we determined that the glutamatergic innervation from the lateral

52 preoptic area of the hypothalamus (LPO) is both pain-responsive and most strongly inhibited by MOR

53 activation. Importantly, we show that activating MORs in this circuit in the absence of pain does not

54 produce reinforcement, suggesting that targeting this circuit could be a significant advance in pain

55 therapeutics.

56

$57 \quad$ Results

58 MOR activation in the LHb produces pain relief but not positive reinforcement

59 To examine the behavioral impact of selective MOR activation in the LHb on ongoing pain, we used the

60 spared nerve injury (SNI) model of persistent neuropathic pain and implanted bilateral cannulae above the

61 LHb in Sprague Dawley rats (Fig. 1a). After recovery from surgery, we evaluated allodynia by measuring

62 mechanical stimulation thresholds with graded von Frey filaments. Bilateral microinjections of the MOR-

63 selective agonist DAMGO $(10 \mu \mathrm{M} ; 300 \mathrm{~nL} /$ hemisphere $)$ into the LHb increased the average hindpaw

64 withdrawal threshold compared to saline microinjections in the same animals, indicating that DAMGO

65 reduced the mechanical allodynia generated by SNI in male rats (Fig. 1b). In contrast, intra-LHb

66 DAMGO microinjections in sham-injured male rats had no effect on mechanical withdrawal thresholds

67 compared to saline (Fig. 1b). Consistent with the rat literature ${ }^{17}$, we did not observe a significant decrease 


\section{Figure 1}

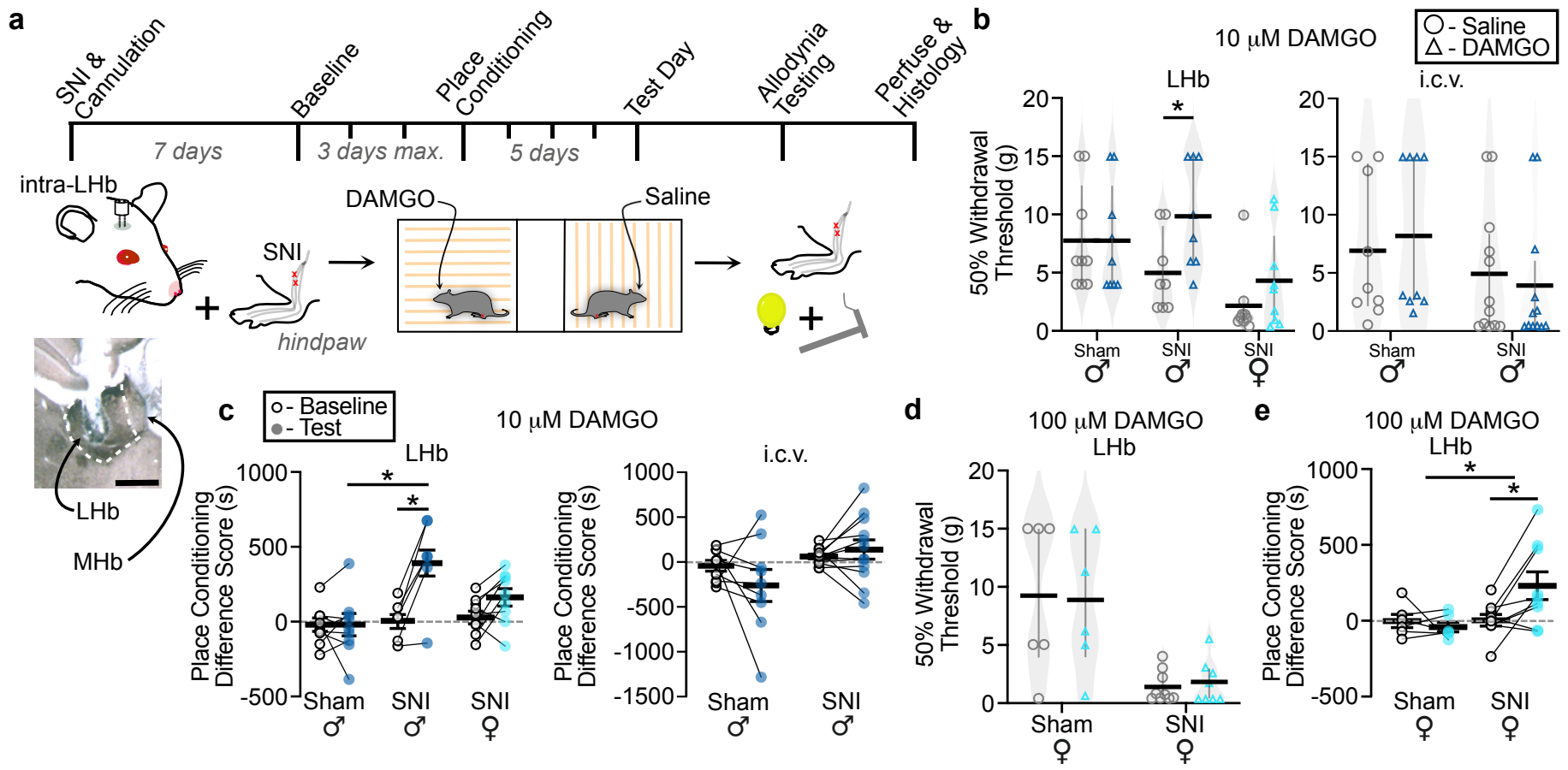

Figure 1. MOR activation in the LHb relieves allodynia and generates negative reinforcement in rats with neuropathic pain. a, Timeline of behavioral experiments. Lower inset, example injection site centered in LHb. Scale bar $=500 \mu \mathrm{m}$. $\mathbf{b}$, (Left) Violin plots of 50\% mechanical withdrawal thresholds following saline or $10 \mu \mathrm{M}$ DAMGO microinjections into the LHb in sham males $(n=9)$, SNI males $(n=8)$, and SNI females $(n=9)$. Sham males: Wilcoxon signed rank test, $V=1.5$, $\mathrm{p}=1$. SNI males: Wilcoxon signed rank test, $\mathrm{V}=2, \mathrm{p}=0.050$. Females trended towards an increase in threshold following DAMGO infusion: Wilcoxon signed rank test, $V=7, p=0.074$. (Right) Mechanical withdrawal thresholds in male rats with i.c.v. cannulae, with SNI $(n=12)$ or sham $(n=9)$ injuries. Sham: Wilcoxon signed rank test, $V=7, p=0.53$. SNI: Wilcoxon signed rank test, $\mathrm{V}=31, \mathrm{p}=0.080$. $\mathbf{c}$, Difference scores before and after conditioning with intracranial microinjections. (Left) Males with SNI developed a preference for the chamber paired with intra-LHb DAMGO. Twoway mixed ANOVA, significant interaction between SNI/sham and baseline/test $\mathrm{F}(1,13)=15.93, \mathrm{p}=0.002$; post hoc effect group on test day adjusted $p=0.01$; paired t-tests, sham adjusted $p=1$; SNI adjusted $p=0.016$. Female SNI animals trended towards a preference for the DAMGO-paired chamber: Paired t-test $p=0.054$. (Right) i.c.v. DAMGO did not produce a preference in sham or SNI male rats: Two-way mixed ANOVA, $F(1,19)=2.239, p=0.15$. $d$, Mechanical withdrawal thresholds in sham $(n=6)$ and SNI $(n=9)$ female rats following $100 \mu$ M DAMGO infusions into the LHb. Sham: Wilcoxon signed rank test, $V=5, p=1$. SNI: Wilcoxon signed rank test, $V=6, p=0.40$. e, Place conditioning of sham and SNI females to intra-LHb $100 \mu \mathrm{M}$ DAMGO vs saline. Females with SNI developed a significant CPP: Twoway mixed ANOVA, significant interaction between SNI/sham and baseline/test $F(1,13)=6.234, p=0.027$; post hoc effect group on test day adjusted $\mathrm{p}=0.07$; paired t-tests, sham adjusted $\mathrm{p}=0.48$; SNI adjusted $\mathrm{p}=0.035$. Means represented by horizontal lines; SEM and quartiles indicated by vertical lines with and without caps, respectively. ${ }^{*} p \leq$ $0.05, * * p<0.01$ 
in withdrawal latency to heat in the Hargreaves test after SNI compared to sham-injured controls, and DAMGO microinjections into the LHb did not alter heat withdrawal latency compared to saline in SNI or in sham animals (Extended Data Fig. 1a). Intra-LHb DAMGO also reversed mechanical allodynia induced by inflammatory pain in the CFA model (Extended Data Fig. 1b-g).

To evaluate whether MOR activation in the LHb influences the affective experience of pain, we used the place conditioning paradigm in the same group of rats. In a three-chamber apparatus, we paired intra-LHb DAMGO microinjections with one chamber and saline microinjections with the opposite side chamber. Rats with SNI developed a significant conditioned place preference (CPP) for the LHb-DAMGO-paired chamber, while sham-injured rats did not prefer either chamber following conditioning (Fig. 1c).

To rule out the potential confound of off-target effects due to DAMGO entering the CSF space via the nearby third ventricle, we microinjected the same solutions intracerebroventricularly (i.c.v.) in male rats with SNI or sham injury. This manipulation did not influence mechanical withdrawal thresholds compared to saline microinjection in either group (Fig. 1b). Furthermore, i.c.v. DAMGO microinjections did not generate a CPP in either SNI or sham animals (Fig. 1c). Therefore, we conclude that the behavioral effects of our DAMGO microinjections were due to actions specifically in the LHb.

We also investigated whether LHb MOR activation had the same effects on allodynia and affective pain in female rats. Using the same microinjection parameters as in male rats, female rats with SNI showed a trend towards reduced mechanical allodynia following DAMGO microinjections into the LHb compared to saline (Fig. 1b). Female rats with SNI also showed a trend towards a preference for the DAMGOpaired chamber (Fig. 1c). As in males, females with SNI displayed no difference in heat withdrawal latency between DAMGO and saline microinjections (Extended Data Fig. 1a). Because female rats may be less sensitive to the analgesic effect of opioids ${ }^{18,19}$, we tested a 10 -fold higher concentration of intraLHb DAMGO $(100 \mu \mathrm{M})$ in a separate cohort of females. This dose was chosen based on studies demonstrating a three-fold reduction in i.c.v. DAMGO efficacy for females in the tail flick test ${ }^{20}$. The 
higher DAMGO dose induced a significant CPP, though still did not reverse the mechanical allodynia in female rats (Fig. 1d, e). Thus, we conclude that LHb MOR activation can reverse the affective experience of pain without raising the baseline threshold for reflex withdrawal in both male and female rats.

\section{MOR synaptic function persists in LHb neurons in animals with chronic pain}

In various CNS regions, chronic pain induces changes in MOR expression and function, including downregulation ${ }^{21-23}$. We previously reported that in naïve male rats MOR activation inhibits glutamate release onto a subset of $\mathrm{LHb}$ neurons and also hyperpolarizes approximately $30 \%$ of $\mathrm{LHb}$ neurons ${ }^{16}$. Here we exampled whether these MOR effects are altered in a persistent pain state. To evaluate postsynaptic MOR function we performed whole cell voltage clamp recordings of LHb neurons from acute brain slices from male rats with SNI and measured DAMGO induced changes to holding current (Fig. 2a, b). These responses did not differ from our observations in naïve rats. We also tested MOR inhibition of glutamatergic electrically-evoked excitatory postsynaptic currents (EPSCs) in LHb neurons from animals with SNI. These responses were also consistent with observations from naïve rats (Fig. 2c, d). We conclude that these actions of MOR on LHb cell bodies and glutamatergic terminals do not change in animals with ongoing pain.

Increased activity in LHb neurons as well as increased glutamatergic synaptic strength onto LHb neurons are associated with aversive behavioral states ${ }^{7,24,25}$. To evaluate this in LHb neurons from animals with SNI, we first measured the paired pulse ratio in the evoked EPSCs, a measure of probability of release. There was no difference in paired pulse ratio between groups (Fig. 2e). We next compared the frequency and magnitude of spontaneous glutamatergic EPSCs (sEPSCs) in animals with SNI to those in naïve animals. Mean sEPSC frequency and amplitude were also similar in LHb neurons from SNI and naïve animals (Fig. 2f, g). Together, these observations suggest a lack of synaptic plasticity induced on glutamatergic inputs to LHb neurons by painful injury. 
Figure 2
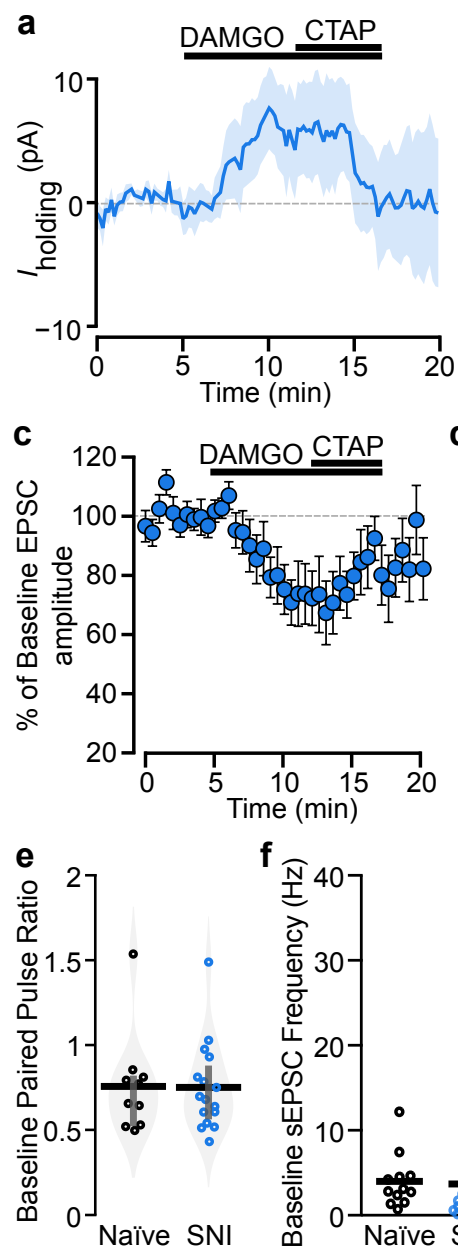

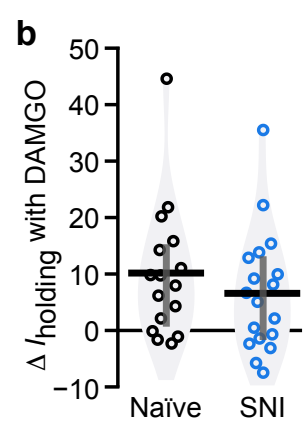

d
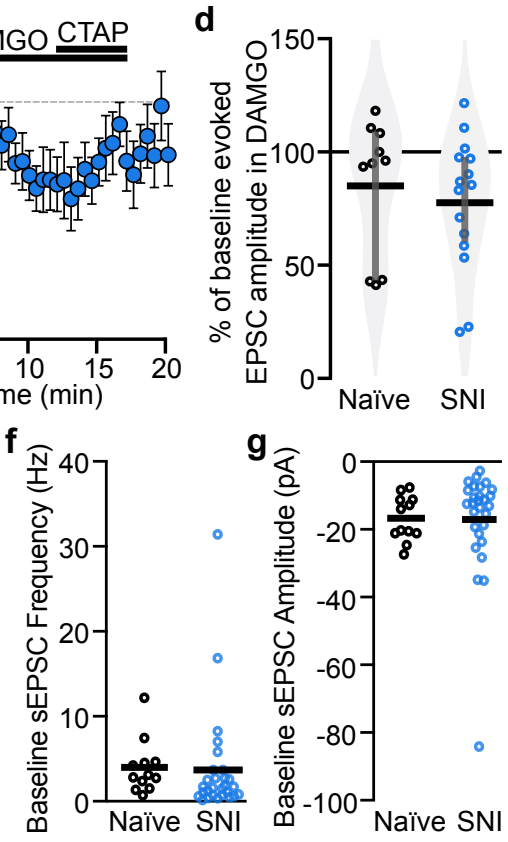

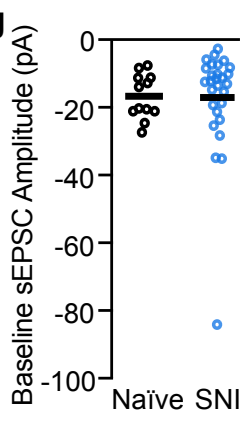

Figure 2. Ongoing pain does not alter MOR agonist synaptic effects or probability of glutamate release in the $L H b$. Responses to the MOR agonist DAMGO were recorded in voltage clamp, $V_{m}=-60$ $\mathrm{mV}$. a, In animals with SNI, a subset of neurons responded to bath application of the MOR agonist DAMGO (500 nM) with a time locked outward current that was reversed with the MOR selective antagonist CTAP (500 nM). b, Postsynaptic holding current changes in response to bath application of DAMGO were not different between neurons from naïve and SNI rats: Unpaired t-test $\mathrm{df}=32, \mathrm{t}=$ $0.892, \mathrm{p}=0.379$. c, In rats with SNI, DAMGO (500 $\mathrm{nM})$ induced a time locked inhibition of electrically evoked glutamatergic EPSCs, and CTAP (500 $\mathrm{nM}$ ) reversed the effect. d, This DAMGO inhibition of electrically evoked EPSC amplitude was not different between $\mathrm{LHb}$ neurons from naïve and SNI rats: Unpaired t-test $\mathrm{df}=21, \mathrm{t}=0.137, \mathrm{p}$ $=0.89$. Baseline probability of release at glutamatergic synapses was similar between LHb neurons in naïve and SNI rats as measured by (e) paired pulse ratio (P2/P1) of electrically stimulated EPSCs (50 ms inter stimulus interval): Unpaired t-test, $\mathrm{df}=24, \mathrm{t}=0.102, \mathrm{p}=0.92$; (f) baseline spontaneous EPSC frequency: Unpaired t-test unequal variances, $\mathrm{df}=37, \mathrm{t}=-0.17, \mathrm{p}=0.87$; and $(\mathrm{g})$ spontaneous EPSC amplitude: Unpaired t-test unequal variances, $\mathrm{df}=38, \mathrm{t}=-0.09, \mathrm{p}=$ 0.93 . Data from naïve rats previously published in $^{16}$. 
$\mathrm{LHb}$ neuron firing activity increases with acute noxious stimulation, and an increase in ongoing firing frequency is during aversive behavioral states ${ }^{2,3,7}$. In ongoing pain, since we did not observe evidence for changes in glutamatergic synaptic strength, such increases in firing may be driven by greater activity in the glutamatergic axons innervating LHb neurons. Therefore, we hypothesized that the intra- $\mathrm{LHb}$ DAMGO-induced behavioral effects that we observed in injured animals were due to MOR inhibition of glutamatergic axon terminals, thus decreasing the aversive excitatory drive onto the LHb neurons. Since MOR activation only inhibits glutamatergic inputs onto a subset of LHb neurons ${ }^{16}$, MORs might be preferentially expressed on specific afferent inputs. Prior work characterizing direct functional synaptic connections to the $\mathrm{LHb}$ is limited to mice ${ }^{26}$, therefore first we sought to confirm these functional connections in the rat. We investigated inputs from the entopeduncular nucleus (EPN), lateral preoptic area of the hypothalamus (LPO), and ventral tegmental area (VTA) because stimulating glutamatergic LHb inputs from these sources has been shown to be aversive $e^{24,27,28}$. We also investigated inputs from the LH and ACC that are strongly implicated in pain processing ${ }^{29}$ and the VP because stimulating glutamatergic VP neurons increases the firing rate of LHb neurons ${ }^{30}$. We injected AAV2-hSynhChR2(H134R)-mCherry into one of these six regions in order to express channelrhodopsin (ChR2) in these different input populations (Fig. 3a). We then made whole cell recordings in LHb neurons and measured light-evoked synaptic inputs while blind to injection site. ChR2 was activated by an LED $(\lambda=$ 473) coupled to an optic fiber placed approximately $100 \mu \mathrm{m}$ from the recorded cell. Post synaptic currents (PSCs) were measured in response to paired light pulses (1 or $5 \mathrm{~ms}, 50 \mathrm{~ms}$ inter stimulus interval) at holding potentials of $-60 \mathrm{mV}$ and $-40 \mathrm{mV}$ to probe for EPSCs and $\mathrm{GABA}_{\mathrm{A}} \mathrm{R}$ mediated inhibitory PSCs (IPSCs) in each cell, respectively. Roughly similar proportions of LHb neurons received synaptic input from each of these targets, with the exception of the ACC, where we did not detect any fast PSC connections (Fig. 3b, topographical distribution of connected neurons in Extended Data Fig. 2). The absence of a functional synaptic input from the ACC to the LHb was surprising, as both anterograde ${ }^{10,12}$ 
Figure 3

a

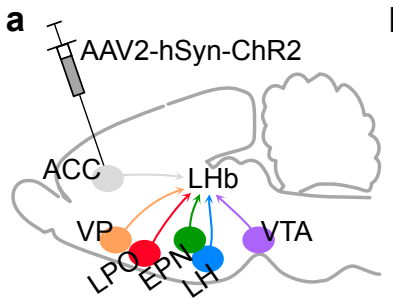

C

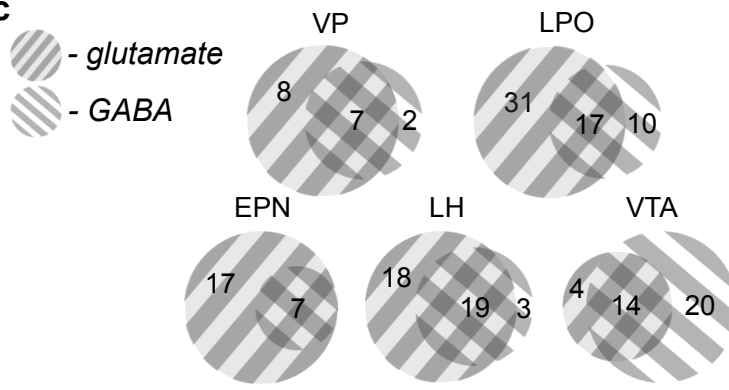

d
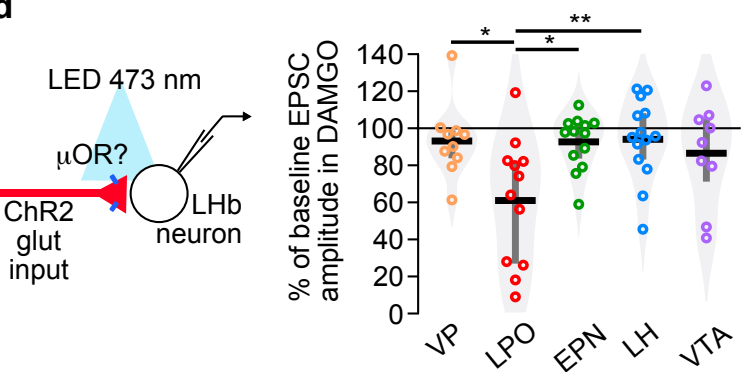

e
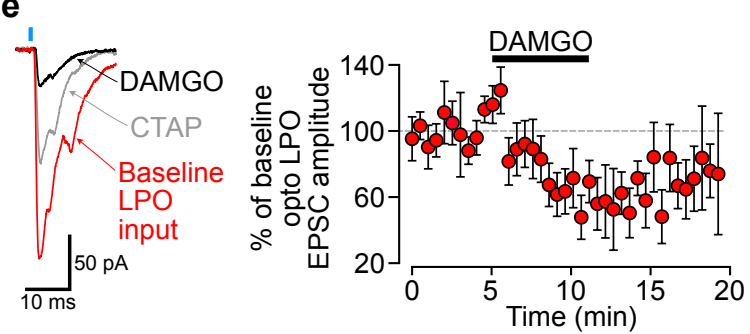

f
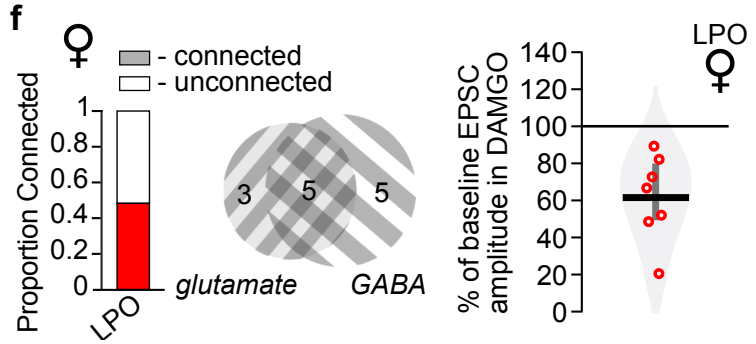

Figure 3. Glutamatergic inputs to the LHb from the LPO are inhibited by MOR activation. a, Schematic diagram of AAV2-hSynhChR2(H134R)-mCherry injection targets which project to the LHb. b, Proportions of $\mathrm{LHb}$ neurons receiving functional synaptic connections from six input regions measured with optogenetic stimulation of terminals expressing ChR2. c, Among connected neurons, venn diagrams depict the numbers of $\mathrm{LHb}$ cells receiving optically stimulated glutamatergic and/or GABAergic synaptic input. d, (Left) Experiment design: DAMGO (500 nM) was applied during optical synaptic stimulation experiments to probe for functional MOR presynaptic control of glutamate release from specific inputs. (Right) Change from baseline amplitude of optically-evoked EPSCs during DAMGO application. EPSCs from LPO terminal stimulation were most strongly inhibited by MOR activation: One-way ANOVA, $\mathrm{df}=4, \mathrm{~F}=4.11, \mathrm{p}=0.0057$ followed by Tukey HSD pairwise comparisons. e, (Left) Representative traces of optically-evoked EPSCs in an LHb neuron from a rat with ChR2 expressed in inputs from the LPO. DAMGO decreased the amplitude of light-evoked EPSC, which was partially reversed by the selective MOR antagonist CTAP (500 nM). Rise time was $<2$ $\mathrm{ms}$, indicative of a monosynaptic response. (Right) Summary time course of the DAMGO inhibition across all optically-evoked LPOLHb EPSCs $(n=12)$. f, LPO inputs to the LHb in female rats (left) had a similar overall connectivity rate but (middle) more GABAergic connections than glutamate connections. (Right) DAMGO inhibited light-evoked glutamatergic synaptic inputs from the LPO to the LHb in female rats as well. 
and retrograde tracers ${ }^{11}$ have previously demonstrated modest inputs. Moreover, the ACC is extensively implicated in behavioral responses to pain and MOR-agonist induced pain relief ${ }^{31,32}$. As a secondary measure of the strength of the innervation, we performed a systematic evaluation of the potential connection using ChR2 as an anterograde tracer, making large injections of AAV2-hSyn-hChR2(H134R)mCherry throughout the anteroposterior range of the ACC (Extended Data Fig. 3a). This tracing revealed extensive innervation of the nearby mediodorsal thalamus (MDL), but minimal stereologically-quantified labeling in the LHb (Extended Data Fig. 3b, c). Therefore, while we cannot completely rule out a functional input from the ACC to the $\mathrm{LHb}$, the innervation is extremely small compared to the other sources of input to the $\mathrm{LHb}$ investigated here.

For each brain region from which fast synaptic PSCs were detected in the LHb, both glutamate and GABA inputs were observed in varying proportions (Fig. 3c). Interestingly, while for each input there were individual $\mathrm{LHb}$ neurons that received both glutamate and GABA synaptic connections, for each input more than half of the connected LHb neurons received just one type of fast PSC, in varying proportions. The VTA was the only input where more LHb neurons received GABAergic synaptic connections than glutamatergic synaptic connections. Among observed synaptic connections, a wide range of EPSC and IPSC amplitudes were observed for most of the inputs, except for the glutamatergic inputs from the VTA that were consistently small (Extended Data Fig. 4a,c). The delay to light evoked EPSC onset also varied across input source, with LH inputs having the shortest mean latency (Extended Data Fig. 4b).

The nature of local LHb neural connections will also impact the circuit's response to MOR activation. There are strong local glutamatergic connections within the $\mathrm{LHb}^{16,33}$, but there is recent evidence both for $^{34,35}$ and against $\mathrm{t}^{36,37}$ the existence of local GABA interneurons. As we only observed somatodendritic MOR responses in a subset of $\mathrm{LHb}$ neurons, evidence for GABAergic interneurons in the $\mathrm{LHb}$ would impact our model of how MOR activation modulates LHb neural activity, if such interneurons preferentially express the MOR. In the rat, a small number of GAD1 positive neurons are present in the 
lateral $\mathrm{LHb}$, though these neurons do not co-express vesicular GABA transporter ${ }^{36}$, the protein required for loading GABA into synaptic vesicles. In order to detect functional local GABAergic connections within the rat LHb, we injected AAV2-hSyn-hChR2(H134R)-mCherry into the LHb and recorded from LHb neurons. Because some recorded neurons expressed $\mathrm{ChR} 2$, we measured light responses before and after application of receptor antagonists in order to isolate the synaptically driven response from the ChR2 mediated currents. Under these conditions we did not observe any light activated local IPSCs in LHb neurons (Extended Data Fig. 5). As expected, many neurons received local glutamatergic inputs (Extended Data Fig. 5). We conclude that there is very limited or no local GABAergic interneuron connectivity in the $\mathrm{LHb}$ of adult rats.

\section{MOR activation most strongly inhibits LPO inputs to the LHb}

Next, we tested for functional MOR modulation of the light evoked glutamatergic inputs to LHb neurons from each of the regions characterized above. DAMGO induced the strongest and most consistent inhibitions in the terminals arising from LPO neurons (Fig. 3d, e). On average the inhibition was greater in these LPO inputs than the MOR impact on ESPCs observed from the LH, VTA, VP, or EPN (Fig. 3d). We also tested whether MOR inhibits LPO glutamatergic inputs to the LHb independent of sex; the mean inhibition of glutamate release from LPO terminals to LHb neurons in female rats was equivalent to that observed in males (Fig. 3f). Because of the prevalence of local glutamatergic connections in the LHb ${ }^{16,33}$ (Extended Data Fig. 5) and postsynaptic MOR inhibition of a subset of LHb neurons ${ }^{16}$, we sought to rule out a polysynaptic connection. First, a polysynaptic contribution seems unlikely for all of the glutamatergic inputs reported here because the delay from light pulse onset to EPSC onset was consistently less than $3 \mathrm{~ms}$ (Extended Data Fig. 4b). Second, to directly test isolated monosynaptic connections, we expressed ChR2 in LPO neurons and recorded in the LHb; in neurons with light evoked EPSC responses, we applied tetrodotoxin (TTX, $500 \mathrm{nM})$ and 4-aminopyridine (4 AP; $10 \mu \mathrm{M})$. In 8 of 8 tested neurons the light evoked EPSCs persisted in this monosynaptic signal sparing preparation, and this monosynaptic response was inhibited by DAMGO in all 5 tested neurons (Extended Data Fig. 6). 
Therefore, we conclude that MORs are functionally expressed on LPO terminals that monosynaptically contact LHb neurons, and when these glutamatergic inputs are activated in vivo, DAMGO application should inhibit them.

\section{MOR mRNA is enriched in LHb-projecting LPO neurons}

Multiple basal forebrain structures express high levels of MOR including the VP, medial preoptic area (MPO), horizontal diagonal band (HDB), ventral bed nucleus of the stria terminalis (vBNST), and other regions of the extended amygdala complex $(\mathrm{EAC})^{38}$. Some of these not only express MOR to a greater extent than the LPO, but they also project to the LHb. To further evaluate the specificity of MOR expression in LHb-projecting neurons in the LPO compared to nearby brain regions, we performed in situ hybridization for MOR mRNA (OPRM1) in brain slices from Sprague Dawley rats where the retrograde tracer Fluoro-Gold had been iontophoresed into the LHb (Fig. 4a - c). With this independent approach, the LHb-projecting LPO neurons showed the strongest OPRM1 expression and contained the greatest number of retrogradely labeled FG(+) neurons co-labeled for OPRM1 (112 \pm 9 cells), corresponding to $57.1 \% \pm 4.3 \%$ of all $\mathrm{MOR}(+) / \mathrm{FG}(+)$ neurons in the basal forebrain $(336 / 602$ total cells). Inputs were also observed from the HDB $(23.6 \% \pm 5.9 \%$ of MOR $(+)$ cells; $153 / 602$ total cells $)$, VP $(9.5 \% \pm 3.1 \%$ of MOR(+) cells; $52 / 602$ total cells), MPO (3.0\% $\pm 1.1 \%$ of MOR $(+)$ cells; $19 / 602$ total cells), vBNST $(3.7 \% \pm 1.2 \%$ of MOR $(+)$ cells; $23 / 602$ total cells $)$ and $\mathrm{EAC}(3.0 \% \pm 2.1 \% \mathrm{MOR}(+)$ cells; $19 / 602$ total cells; Fig. 4d - f). Overall, these anatomical data are highly consistent with our electrophysiology results, supporting the conclusion that the LPO projection to the LHb is strongly regulated by MORs.

\section{Noxious stimulation activates glutamatergic LHb-projecting LPO neurons}

We next tested whether glutamatergic LHb-projecting LPO neurons are activated by noxious stimulation and whether ongoing pain alters this response. We expressed the calcium indicator GCaMP6m in LHbprojecting LPO neurons using a Cre-dependent, retrograde viral construct HSV-hEF1 $\alpha$-LS1L-GCaMP6m injected to the LHb of VGluT2::Cre mice (Fig. 5a). We implanted optic fibers above the LPO in control 


\section{Figure 4}

a

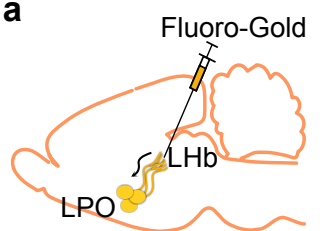

b

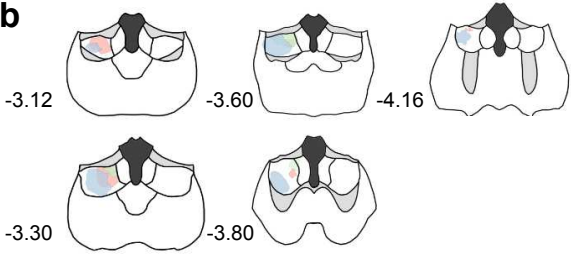
$-3.80$

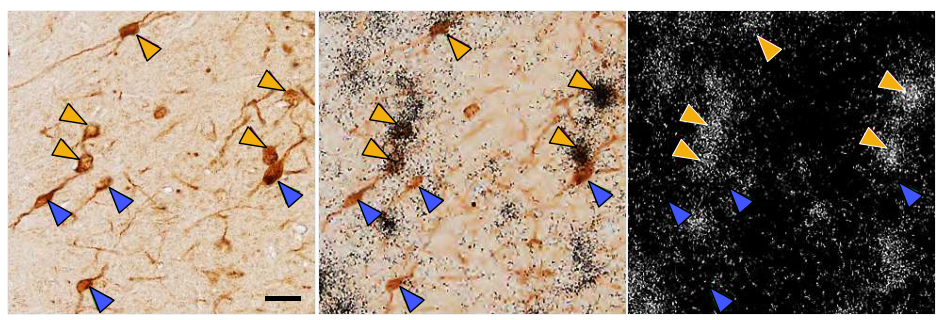

d

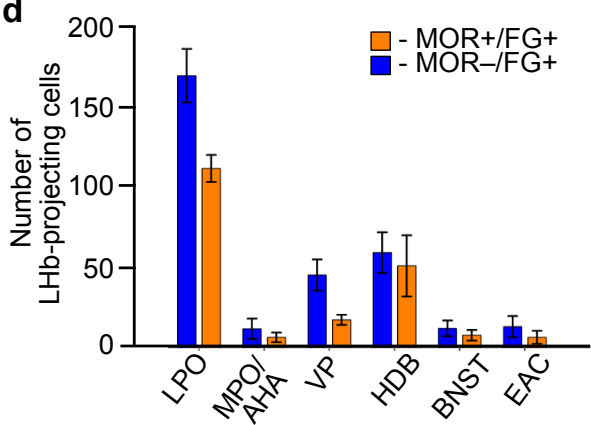

e Proportion of MOR+ Inputs to $\mathrm{LHb}$

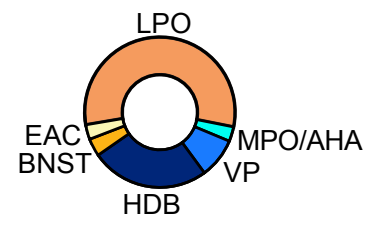

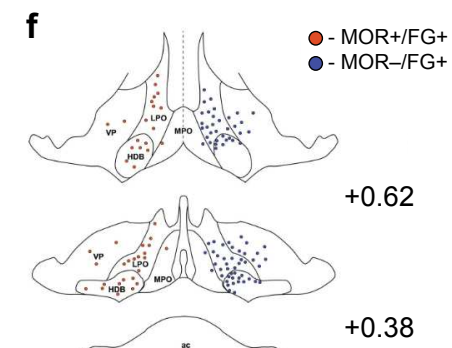

$+0.14$
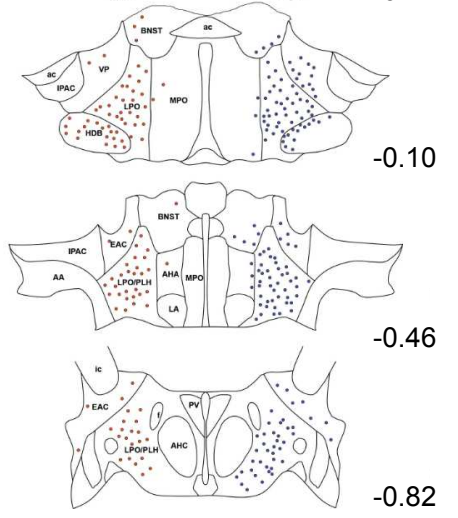

Figure 4. LPO inputs to the LHb highly express MOR $m R N A$. a, Schematic of unilateral iontophoretic deposition of the retrograde tracer FG into the $\mathrm{LHb}$. b, FG injection sites in $\mathrm{LHb}(\mathrm{n}=3$ rats). $\mathbf{c}$, (Left and middle) Representative brightfield images of FG-positive/MOR-positive somas (yellow arrowhead) and FG-positive/MOR-negative somas (blue arrowhead) in the LPO. Scale bar $=20 \mu \mathrm{m}$. (Right) Darkfield image of in situ hybridization localization for MOR mRNA (OPRM1). d, Number of MOR-positive/FG-positive (yellow) and MOR-negative/FG-positive (blue) cells in various basal forebrain regions. e, Donut plot depicting proportions of total MOR-positive inputs to the $\mathrm{LHb}$ from various basal forebrain regions. f, Locations of FG-positive/MOR-positive (red) and FG-positive/MOR-negative (blue) somata throughout basal forebrain. Co-labeled cells are separated from the singly labelled population for display purposes. 


\section{Figure 5}

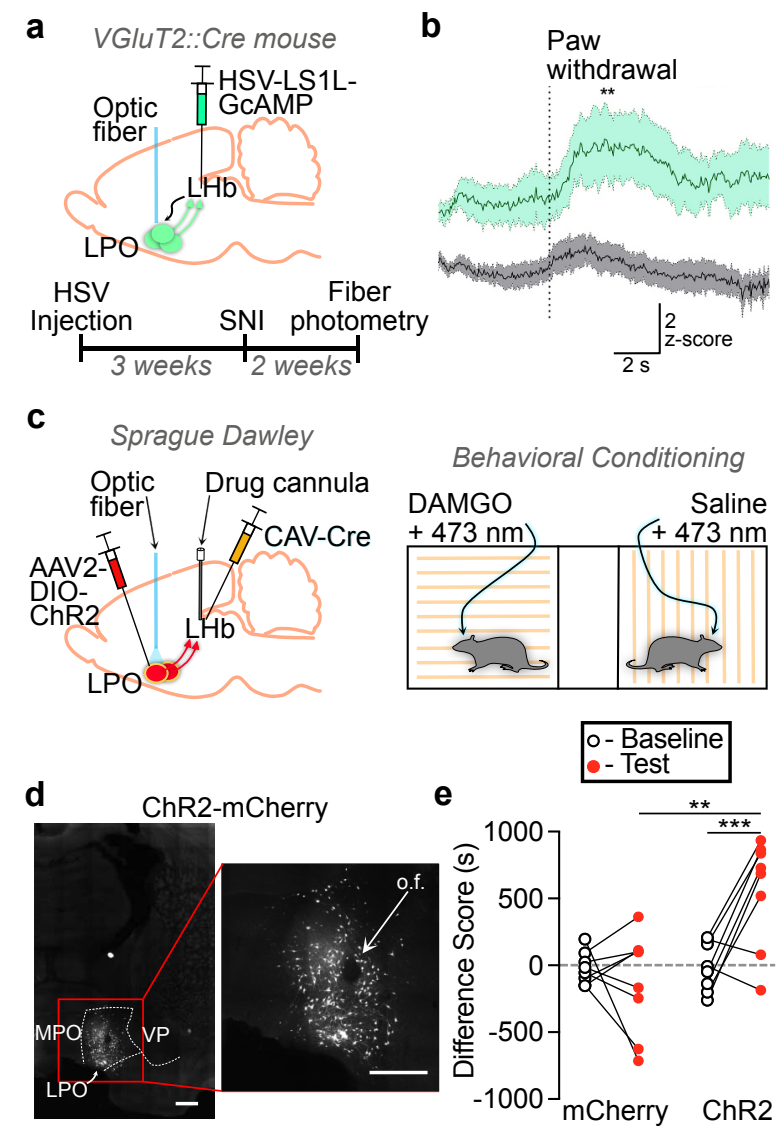

Figure 5. $L H b$ MOR activation relieves aversiveness of glutamatergic LPO input to the LHb. a, (Top) Schematic diagram of HSV-hEF1 $\alpha-$ LS1L-GCaMP6m injection into the LHb of VGluT2::Cre mice, to induce expression of GCaMP6m in glutamatergic LPO neurons innervating the $\mathrm{LHb}$, and optic fiber implant for fiber photometry imaging in the LHb-projecting LPO neurons. (Bottom) Experimental timeline. $\mathbf{b}$, Mice with SNI $(n=5)$ exhibited significantly larger changes in GCaMP6m fluorescence time-locked to paw withdrawal in response to thermal stimulation in the Hargreaves apparatus compared to sham controls $(\mathrm{n}=9)$ : Two-way ANOVA, $\mathrm{F}(1,12)=5.439, \mathrm{p}=$ 0.038; Holm-Sidak post-hoc test, $\mathrm{p}=0.0074$. c, (Left) Schematic diagram of dual virus injections to express ChR2-mCherry or mCherry selectively in LPO neurons that project to the $\mathrm{LHb}$, optic fiber implantation targeting the LPO cell bodies, and cannula implantation targeting the $\mathrm{LHb}$ for experiments in male rats. (Right) Rats received bilateral blue light stimulation of the LPO during all conditioning sessions and in both chambers, sides paired with intra$\mathrm{LHb}$ saline or DAMGO. d, Representative images of Cre-dependent ChR2-mCherry fluorescence in LPO cell bodies. (Left) Scale bar $=$ $250 \mu \mathrm{m}$. (Right) Lesion from optic fiber ("o.f.") implant amidst ChR2-mCherry-expressing cell bodies. Scale bar $=50 \mu \mathrm{m}$. e, Only animals with active ChR2 $(n=8)$, but not mCherry controls $(n=8)$, developed a preference for the intra-LHb DAMGO paired side of the place conditioning apparatus. Two-way mixed ANOVA, significant interaction between ChR2-mCherry/mCherry and baseline/test $F(1,14)=9.982, p=0.007$; post hoc effect group on test day adjusted $\mathrm{p}=0.006$; paired t-tests, mCherry adjusted $\mathrm{p}=0.427$; ChR2-mCherry adjusted $\mathrm{p}=$ 0.0027. $* * \mathrm{p}<0.01, * * * \mathrm{p}<0.005$ 
and SNI mice and performed fiber photometry prior to and during the Hargreaves task. On average,

GCaMP6m signal increased as paw withdrawal commenced, and this response was greatly potentiated in mice with SNI (Fig. 5b), who also displayed shorter withdrawal latencies to heat (Extended Data Fig. 7). Thus, glutamatergic LPO neurons that project to the $\mathrm{LHb}$ are activated in response to noxious peripheral stimulation, and the magnitude of activation is higher during ongoing pain.

\section{LHb MOR activation blocks the aversiveness of LPO-LHb stimulation}

Because LHb-projecting LPO glutamatergic neurons are activated by noxious stimulation, stimulating these terminals in the $\mathrm{LHb}$ is aversive $\mathrm{e}^{28}$, and MOR activation inhibits these terminals in the LHb, we hypothesized that the aversiveness produced by stimulating the LHb-projecting LPO neurons should be reduced by MOR agonist injection into the $\mathrm{LHb}$. To selectively express ChR2 in LHb-projecting LPO neurons, we used an intersectional viral approach in rats, injecting the retrograde CAV-Cre into the $\mathrm{LHb}$ and AAV2-EF1 $\alpha$-DIO-hChR2(H134R)-mCherry (or AAV2-EF1 $\alpha$-DIO-mCherry for controls) into the LPO bilaterally (Fig. 5c,d). Bilateral optic fibers were subsequently implanted above the LPO and cannulae were implanted above the LHb (Fig. 5c). During place conditioning sessions, rats received blue light activation (473 nm, $20 \mathrm{~Hz}, 5 \mathrm{~ms}, 10-12 \mathrm{~mW}$ ) of the LHb-projecting LPO neurons in both environments of the place conditioning apparatus; chambers were paired with either intra-LHb DAMGO or saline (Fig. 5c). Rats with ChR2 expression developed a CPP for the chamber associated with DAMGO, while control mCherry expressing animals did not (Fig. 5e). Therefore, MOR activation in the $\mathrm{LHb}$ blocked the aversiveness of stimulating the LPO input to the LHb without producing positive reinforcement in the absence of input stimulation.

\section{Discussion}

Here we identified a circuit that can be targeted by MOR agonists to relieve the aversiveness of ongoing pain but does not produce reward in pain free rodents. MOR agonist action in the LHb was sufficient to 
reverse injury-induced allodynia and to produce a CPP, decreasing both sensory and affective pain responses, respectively. Importantly, sham-injured animals did not develop a CPP, indicating that MOR activation in the $\mathrm{LHb}$ does not produce positive reinforcement in the absence of pain. Unexpectedly, rather than inputs from brain regions previously established as mediating pain and opioid-induced pain relief, it is the glutamatergic inputs from the LPO that we demonstrate here are preferentially controlled by $\mathrm{LHb}$ MOR activation. We found that the aversiveness produced by optogenetic activation of LHbprojecting LPO neurons is blocked by MOR agonist microinjection into the $\mathrm{LHb}$, showing that a MOR inhibitory action on this circuit is sufficient to relieve pain. Together, these experiments show that MOR activation in the LHb can generate negative reinforcement via pain relief, but not positive reinforcement in the absence of noxious stimuli.

\section{The LHb in pain and relief}

The LHb plays a role in the perception of noxious stimuli in injury and depression model ${ }^{39,40}$, and most LHb neurons fire more in aversive behavioral states and in response to noxious stimuli ${ }^{2,3}$ Bilateral lesions of the $\mathrm{LHb}$ decrease allodynia in the chronic constriction ischemia model of neuropathic pain in rats ${ }^{41}$, supporting the notion that signaling through the LHb contributes to injury-induced mechanical allodynia. Chemogenetic inhibition of the LHb relieves thermal hyperalgesia in animals undergoing alcohol withdrawa ${ }^{39}$. We found that MOR activation in the $\mathrm{LHb}$ reverses mechanical allodynia in a model of neuropathic pain in both males and females. Together these observations support our conclusion that MOR activation impacts the behavioral state of the animal by decreasing LHb neural activity in vivo. We previously found two potential mechanisms by which MOR activation could inhibit LHb neural activity in animals with ongoing pain: presynaptically inhibiting glutamate release and postsynaptically driving an outward current in a subset of LHb neurons ${ }^{16}$. Here we found that intra-LHb MOR activation induces negative reinforcement in both male and female rats with ongoing pain. Yet sham controls (male or female) did not develop a CPP to intra-LHb DAMGO. Because the behavioral effect of MOR activation 
was specific to injured rats we hypothesized that increased glutamatergic drive to the $\mathrm{LHb}$, in an input that is MOR sensitive, is a key element in the LHb circuit dynamics involved in pain and relief.

The vast majority of $\mathrm{LHb}$ neurons are glutamatergic, and there is anatomical evidence for dense boutonlike structures arising from local $\mathrm{LHb}$ neurons ${ }^{42}$. This local feed forward connectivity may distribute a specific excitatory $\mathrm{LHb}$ input across the various $\mathrm{LHb}$ projections that include the dorsal raphe, ventrolateral PAG, MDL, centromedian thalamus, LH, RMTg, and VTA ${ }^{9,13,14,43}$. Local connectivity has also been observed with functional assays, including that TTX application decreases the frequency of sEPSC and sEPSP events in a subset of LHb neurons ${ }^{16,33}$ and that optogenetic activation of LHb neurons induces glutamatergic EPSCs (Extended Data Fig. 5). This feed forward circuit enables the distribution of an afferent excitatory signal across $\mathrm{LHb}$ neurons, and it raises the possibility that inhibition of such an input by a MOR agonist will decrease activity in $\mathrm{LHb}$ projection neurons, even those that are not directly innervated by the excited pathway. Therefore, it is possible that MOR inhibition of one specific input, such as the LPO, can decrease the excitatory drive onto many efferent LHb projections.

We characterized the functional glutamatergic connections from a variety of brain regions to the LHb in rats; our findings were largely consistent with prior reports utilizing similar techniques in mice. Still, we note that outcomes of these experiments are dependent on the types and number of neurons that express ChR2 following the virus injections and are limited to the geometry of an injection site. We detected the strongest glutamatergic inputs from the LH and EPN, while glutamatergic synaptic responses from the VTA were quite small. We found no functional inputs from the ACC, and in a systematic anatomical analysis we detected very few afferent fibers from the ACC in the LHb. Of the detected glutamatergic inputs, we expected brain regions associated with pain perception and pain relief, such as the LH and VTA, to be more strongly modulated by MOR activation. However, we instead found MOR mRNA and function were clearly enriched in LPO inputs to the LHb. 


\section{The LPO: a brain region contributing to pain perception}

286

287

288

289

290

291

292

293

294

Because $\mathrm{LHb}$ neurons generally fire more in response to noxious stimuli, and optogenetic stimulation of various glutamatergic inputs to the LHb is uniformly aversive $\mathrm{e}^{25,27,28,44,45}$, we hypothesized that a glutamatergic input to the $\mathrm{LHb}$ transmits the pain signal. Further, aversive stressors lead to an increase in the ratio of excitatory glutamatergic to inhibitory GABAergic in synaptic input to LHb neurons $\mathrm{s}^{44,45}$, and restoration of this ratio is associated with relief of aversive states such as foot shock-induced learned helplessness ${ }^{45}$ and cocaine withdrawal ${ }^{44}$. Therefore, a pharmacological manipulation that decreases the excitatory drive onto LHb neurons should also relieve aversive states. Because we found that DAMGO in the LHb generates CPP only in animals with ongoing pain, our data suggest a MOR-sensitive glutamatergic input to the $\mathrm{LHb}$ is active during pain and relatively inactive in the absence of pain.

The LPO projection to the LHb is composed of neurons releasing either glutamate or GABA; these neurons do not co-release glutamate and $\mathrm{GABA}^{28}$, unlike other $\mathrm{LHb}_{\text {inpus }}{ }^{46}$. Optogenetic activation of LPO glutamate projections to the LHb is aversive ${ }^{28}$, and here we posited that this activation mimics an ongoing pain signal. We were able to relieve the aversiveness of activation of this connection with a MOR agonist in the LHb. While a causal role for the LPO in pain perception is unexplored to date, anterograde $^{47}$ and retrograde ${ }^{48}$ tracing has shown a direct input to the LPO from the spinal cord. Also, injections of the pro-inflammatory cytokine IL-1ß into the LPO induces hyperalgesia, indicating LPO participation in a nociception circuit ${ }^{49}$. Painful stimuli such as subcutaneous formalin injections, mild electric shock and tail pinch also increase firing in some LPO neurons ${ }^{50,51}$. Here we show that a major output for LPO pain signals is to the LHb.

\section{The LPO to LHb circuit: A unique target for analgesia}

Opioids remain the best available clinical analgesics, yet ongoing systemically administered opioids can result in the serious adverse consequences including opioid use disorder and respiratory depression ${ }^{52}$. Opioid-induced positive reinforcement and euphoria, combined with the development of dependence, 
underlie opioid abuse liability. To date, there is little clinical evidence that the analgesic and euphoric effects of opioids can be decoupled in humans, yet this possibility is a potential pathway to improve therapies for pain. Here we have identified a key circuit whose modulation relieves pain but does not generate reward in the absence of pain. This dissociation between negative and positive reinforcement provides a neural target to achieve pain relief without promoting substance use disorder. Is it possible to activate MORs in the $\mathrm{LHb}$ but not in a reward circuit?

There is some preclinical evidence that this dissociation is possible by developing MOR ligands with the appropriate opioid pharmacology. For example, a novel, cyclized, stabilized MOR selective agonist based on endomorphin I produces pain relief but not reward ${ }^{53}$. An alternative approach would be to target a different receptor with high expression levels in this circuit in a way that would decrease LHb neural activity. Relevant to this approach, mRNA expression for a selection of orphan G-protein coupled receptors (GPCRs) is enriched in the habenula ${ }^{37,54,55}$. One such receptor is GPR151, and LHb neurons containing GPR151 receive input from the $\mathrm{LPO}^{56}$. Such directed strategies present a range of new anatomic and molecular targets for pain therapy. By identifying the LPO-LHb connection as a site able to provide relief from neuropathic pain and injury-induced allodynia, we have discovered a unique circuit that may achieve effective opioid-mediated pain relief independent of the drug's addictive properties.

\section{Online Methods}

\section{Animals}

All experiments were performed in accordance to the guidelines of the National Institutes of Health Guide for the Care and Use of Laboratory Animals, and the Institutional Animal Care and Use Committees (IACUC) at the University of California, San Francisco, the National Institute on Drug Abuse (NIDA), and Rutgers University. 
Male and female Sprague Dawley rats were obtained from Charles River Laboratories. Rats were allowed access to food and water ad libitum and maintained on a 12h:12h light/dark cycle. Rats used in behavioral and in situ hybridization studies were housed under reverse light/dark cycle conditions. Rats were group housed until they underwent surgery, after which they were singly housed.

Male and female VGluT2::Cre mice were bred from mice obtained from the Jackson Laboratory (Jax \# 016963). Mice were allowed access to food and water ad libitum and maintained on a $12 \mathrm{~h}: 12 \mathrm{~h}$ light/dark cycle with lights on at 7 AM. Mice were always housed in groups of 2-5.

\section{Viral constructs}

AAV2-hSyn-hChR2(H134R)-mCherry (titer: 2.9e+12), AAV2-hSyn-mCherry (titer: 4.7e+12), and AAV2-EF1 $\alpha$-DIO-ChR2-mCherry (titer: 5.1e+12) were purchased from the University of North Carolina Vector Core with available stock constructs from the laboratory of K. Deisseroth at Stanford University. CAV-Cre (titer: 2.5e+12) was purchased from Montpelier University, France. HSV-hEF1 $\alpha-G C a M P 6 m$ (titer: 5e+9) was purchased from the Gene Delivery Technology Core at Massachusetts General Hospital.

\section{Stereotaxic injections}

Rats weighing 275-300 g were anesthetized with 3-5\% isoflurane (Henry Schein) via inhalation and secured in a stereotaxic frame. Bilateral craniotomies were created with a dental drill above the injection site. For electrophysiology experiments, injections of AAV2-hSyn-hChR2(H134R)-mCherry were made bilaterally into the LPO (-0.3 mm anteroposterior (AP), $\pm 1.4 \mathrm{~mm}$ mediolateral (ML), $-8.4 \mathrm{~mm}$ dorsoventral (DV)), VP (-0.24 mm AP, $\pm 2.6 \mathrm{~mm} \mathrm{ML,} \mathrm{-7.8} \mathrm{mm} \mathrm{DV),} \mathrm{EPN} \mathrm{(-2.4} \mathrm{mm} \mathrm{AP,} \pm 3.0 \mathrm{~mm} \mathrm{ML},-$ 7.0 mm DV), LH (-2.6 mm AP, $\pm 1.7 \mathrm{~mm} \mathrm{ML},-8.2 \mathrm{~mm}$ DV), VTA (-5.8 mm AP, $\pm 0.5 \mathrm{~mm} \mathrm{ML},-8.5 \mathrm{~mm}$ $\mathrm{DV})$, anterior $\mathrm{ACC}(+2.2 \mathrm{~mm} \mathrm{AP}, \pm 0.6 \mathrm{~mm} \mathrm{ML},-2.6 \mathrm{~mm} \mathrm{DV})$, or posterior ACC $(+1.7 \mathrm{AP}, \pm 0.6 \mathrm{~mm}$ ML, -2.6 mm DV) using a Nanoject II (Drummond Scientific, Broomall, PA). A volume of $\sim 500-830 \mathrm{~nL}$ was injected per hemisphere over a period of $4.5 \mathrm{~min}$. The glass injector tip was left in place for at least 2 
additional minutes before slow withdrawal to prevent backflow and infection of tissue dorsal to the injection target.

For ChR2 behavior experiments, AAV2-EF1 $\alpha$-DIO-hChR2(H134R)-mCherry or AAV2-EF1 $\alpha$-DIOmCherry were injected into the LPO as above and CAV-Cre was injected bilaterally into the LHb (-3.7 $\mathrm{mm}$ AP, $\pm 0.65 \mathrm{~mm} \mathrm{ML},-5.4 \mathrm{~mm} \mathrm{DV}$ ) with a microinjector connected via polypropylene tubing to Hamilton syringes controlled by a dual syringe pump (KD pump) guided by a bilateral 33G stainless steel guide cannula (Plastics One).

Rats were treated with subcutaneous Carprofen $(5 \mathrm{mg} / \mathrm{kg}$, Zoetis) and topical 2\% Lidocaine (Phoenix Pharmaceutical, Inc.) during the surgery for pain control. After surgery, animals had access to liquid Tylenol ( 1:40) in their drinking water for 3-5 days or were administered Meloxicam (s.c. $2 \mathrm{mg} / \mathrm{kg}$, Pivetal) once per day for two days.

\section{Cannulation and optic fiber implantation}

Two to four weeks after virus injection, rats slated for behavioral testing underwent a second cranial surgery to implant custom-made $200 \mu \mathrm{m}$ optic fibers at a $5^{\circ}$ angle of rotation in the coronal plane into the bilateral LPO (-0.6 mm AP, $\pm 3.05 \mathrm{~mm} \mathrm{ML},-7.5 \mathrm{~mm} \mathrm{DV})$. For microinjections into the LHb, bilateral guide cannulae were implanted $1 \mathrm{~mm}$ above the $\mathrm{LHb}(-3.7 \mathrm{~mm} \mathrm{AP}, \pm 0.65 \mathrm{~mm} \mathrm{ML},-4.4 \mathrm{~mm} \mathrm{DV})$. A dummy stylet was inserted to maintain patency of the cannulae. Optic fibers and cannulae were anchored with flat point screws and dental cement. For i.c.v. microinjections, unilateral cannulae were implanted into the right lateral ventricle (-1.0 $\mathrm{mm} \mathrm{AP},+1.5 \mathrm{~mm} \mathrm{ML},-3.5 \mathrm{~mm} \mathrm{DV})$.

Analgesia during surgery and recovery was administered as described above. Animals were allowed to recover for 1-2 weeks prior to behavioral testing. All virus injections, optic fiber and cannulae placements were histologically verified postmortem based on the standard rat brain atlas ${ }^{57}$. 
378

379

380

381

382

383

384

385

386

Spared nerve injury (SNI) of the sciatic nerve branch was performed to model chronic neuropathic pain ${ }^{17}$. Under isoflurane anesthesia, a 2-cm skin incision was made over the left hindlimb. The biceps femoris muscle was blunt dissected to expose the branches of the sciatic nerve. The common peroneal and tibial nerves were ligated with 5.0 silk surgical suture and transected distally, with sparing of the sural nerve branch. For sham procedures, a skin incision was made and biceps femoris muscle was exposed without dissection. The overlying skin was closed with a monocryl suture. Animals were allowed seven days to recover from surgery prior to behavioral testing. A majority of animals demonstrated decreased mechanical withdrawal thresholds after SNI; those that did not demonstrate allodynia were not used in further experiments.

\section{Inflammatory pain model}

Peripheral inflammation was induced using Complete Freund's Adjuvant (CFA; Sigma Life Science). Under isofluorane anesthesia, a 1:1 emulsion of CFA and sterile saline $(150 \mu \mathrm{L})$ was injected into the footpad of the rat's left hindpaw with a $27 \mathrm{G}$ needle. Sham-injured controls were injected with sterile saline $(150 \mu \mathrm{L})$.

\section{Microinjections}

Rats were lightly restrained in a cloth wrap for intracranial microinjections. A bilateral 33G microinjector (PlasticsOne) that extended $1 \mathrm{~mm}$ ventrally beyond the guide cannula was inserted to target drug delivery into the LHb or i.c.v. Hamilton syringes were driven by a dual syringe pump to infuse either vehicle (phosphate buffered saline, PBS) or DAMGO (10 $\mu \mathrm{M}, 300 \mathrm{~nL} /$ hemisphere administered over $2 \mathrm{~min}$ ). A separate cohort of female rats was microinjected with $100 \mu \mathrm{M}$ DAMGO. 
Standard von Frey sensory assessments were performed as described ${ }^{58}$. Briefly, rats were habituated to sensory testing chambers (Plexiglass boxes with mesh-like flooring) for at least 2 days prior to testing. Behavioral assessments did not begin until exploratory behavior subsided. Testing was completed with eight Touch Test ${ }^{\circledR}$ fibers (North Coast Medical \& Rehabilitation Products, Gilroy, CA, USA) ranging from 0.4 to $15 \mathrm{~g}$. Fibers were pressed perpendicularly to the mid-plantar left hindpaw with sufficient force to cause bending in the fiber and held for 3-4 s. A positive response was noted if the paw was sharply withdrawn. Ambulation was not considered a positive response. When responding was ambiguous, testing was repeated. $50 \%$ withdrawal thresholds were calculated using fiber application in ascending stiffness order or using the up down method.

In behavioral pharmacology experiments rats underwent sensory testing 5 min after counterbalanced saline or DAMGO microinjections on the same day, with at least $4 \mathrm{~h}$ between infusions. Experimenters were blinded to the solution composition during administration and testing.

\section{Hargreaves sensory assessment}

Hargreaves tests were completed in sensory testing chambers with glass flooring. Assessment commenced after rats acclimated to the chamber. Testing was completed using a plantar test analgesia meter (Series 8 Model 390, IITC Life Science, Woodhills, CA, USA). Radiant light was directed toward the mid-plantar left hindpaw until a sharp paw withdrawal response was observed with a $30 \mathrm{~s}$ maximum cutoff. Ambulation was not considered a positive response. At baseline testing, measurements were repeated at different intensity levels until the average withdrawal latency of eight trials was $15 \pm 2 \mathrm{~s}$. Subsequent measurements were performed using this individualized intensity level.

\section{Place conditioning}

Conditioned place preference (CPP) pairings occurred twice daily for four consecutive days following the post-surgery sensory tests with DAMGO and saline. The conditioning apparatus (Med. Associates, 
422 Georgia, VT, USA) was divided into two chambers $(25 \mathrm{~cm} \times 21 \mathrm{~cm} \times 21 \mathrm{~cm})$ with distinct visual

423 (horizontal vs. vertical stripes) and textural (thick vs. thin mesh flooring) cues, separated by a third,

424 smaller gray chamber $(12 \mathrm{~cm} \times 21 \mathrm{~cm}$ x $21 \mathrm{~cm})$. Before conditioning commenced, animals were allowed

425 up to three opportunities to show neutrality across the chambers during 30-minute baseline sessions. Rats

426 that displayed a consistent baseline preference ( $>65 \%$ of time spent in one chamber) were excluded from

427 the study. Rats were pseudorandomly assigned to receive DAMGO in one of the larger chambers, and

428 assignments were counterbalanced for each cohort.

429 During conditioning sessions, microinjections were performed as described above through the intra-LHb

430 cannulae just before the rat was confined to the designated chamber for $30 \mathrm{~min}$. One saline or one

431 DAMGO microinfusion was administered per conditioning session, morning and afternoon pairing

432 sessions were at least $4 \mathrm{~h}$ apart, and the order of administration was alternated on each day of

433 conditioning. On test day, rats were allowed to freely explore the chambers for $30 \mathrm{~min}$ and time spent (s)

434 in each partition was recorded. Difference score was defined as (Time spent in DAMGO-paired chamber)

435 - (Time spent in saline-paired chamber).

436 For ChR2 activation studies, rats were acclimated to handling and attachment of fiber cables to fiber

437 implants in a neutral environment. On procedure days, fiber implants were connected to optic fiber cables

438 attached to a 1x 2 fiber optic rotary joint (Doric Lenses, Quebec, Canada). A laser light source (MBL 473,

439 OEM Laser Systems, East Lansing, MI) was used with light intensity at the end of the output fiber

440 adjusted to $80-120 \mathrm{~mW} / \mathrm{mm}^{2}$. Light stimulation (5 ms pulses at $20 \mathrm{~Hz}$ ) commenced upon placement of

441 rats into the designated chamber of a custom built apparatus on conditioning days. During baseline and

442 testing sessions, the time spent in each chamber was recorded using a webcam and analyzed using Viewer

443 software (Biobserve, Bonn, Germany). 


\section{Brain removal and immunohistochemistry}

445

446

447

448

449

450

451

452

453

454

455

456

457

458

459

460

461

462

463

464

465

466

467

Rats were deeply anesthetized with an intraperitoneal injection of Euthasol $(0.1 \mathrm{mg} / \mathrm{kg}$, Virbac Animal Health, Fort Worth, TX) after $0.3 \mu 1$ of Chicago Sky Blue (in 2\% PBS) was injected through the cannulae to mark injection locations. After becoming unresponsive to noxious stimuli, the rats were transcardially perfused with $400 \mathrm{~mL}$ of saline, followed by $400 \mathrm{~mL}$ of $4 \%$ paraformaldehyde (PFA) in $0.1 \mathrm{M}$ phosphate buffer. The brains were extracted and immersion-fixed in PFA for $2 \mathrm{~h}$ at room temperature (RT), washed two times with PBS to remove excess PFA, and stored in $1 \mathrm{X}$ PBS at $4^{\circ} \mathrm{C}$ until they were sectioned (50 $\mu \mathrm{m}$ ) using a vibratome (Leica VT $1000 \mathrm{~S}$ ). Sections were mounted on slides using VECTASHIELD ${ }^{\circledR}$ mounting medium (Vector Laboratories, Burlingame, CA, USA). Images were taken under a Zeiss Stemi 2000-C (Pleasanton, CA) using an Amscope MD800E running AmScope x64 3.0 Imaging Software.

To label biocytin filled cells after slice electrophysiology recordings, slices were washed three times for 5 min each with PBS (Gibco, Waltham, MA), then blocked with a solution containing: bovine serum albumin $(0.2 \%)$, normal goat serum (5\%) and Tween20 (0.3\%; Sigma-Aldrich, St. Louis, MO) for $2 \mathrm{~h}$ at RT. Slices were incubated in DTAF-streptavidin (1:200; Jackson Immuno Research) diluted in PBS + $0.3 \%$ Tween 20 for $48 \mathrm{~h}$ at $4^{\circ} \mathrm{C}$. After five, 10 -min rinses, brain slices were mounted onto glass slides as above and imaged using a Zeiss Axioskop upright microscope (2.5X, NA $=0.075$ or Plan Apochromat $20 \mathrm{X}, \mathrm{NA}=0.75)$.

\section{Electrophysiology}

Rats were deeply anesthetized with isoflurane, decapitated, and brains were quickly removed into ice-cold artificial cerebrospinal fluid (aCSF) consisting of (in $\mathrm{mM}$ ): $119 \mathrm{NaCl}, 2.5 \mathrm{KCl}, 1.0 \mathrm{NaH}_{2} \mathrm{PO}_{4}, 26.2$

$\mathrm{NaHCO}_{3}, 11$ glucose, $1.3 \mathrm{MgSO}_{4}, 2.5 \mathrm{CaCl}_{2}$, saturated with $95 \% \mathrm{O}_{2}-5 \% \mathrm{CO}_{2}$, with a measured osmolarity 310-320 mOsm/L. Two hundred $\mu \mathrm{m}$ coronal sections through the LHb were cut with a Leica VT $1000 \mathrm{~S}$ vibratome. Slices were incubated in oxygenated aCSF at $33{ }^{\circ} \mathrm{C}$ and allowed to recover for at least one hour. A single slice was placed in the recording chamber and continuously superfused at a rate of 
$2 \mathrm{~mL} / \mathrm{min}$ with oxygenated aCSF. Neurons were visualized with an upright microscope (Zeiss

AxioExaminer.D1) equipped with infrared-differential interference contrast, Dodt optics, and fluorescent

illumination. Whole cell recordings were made at $34^{\circ} \mathrm{C}$ using borosilicate glass microelectrodes (3-5 M $\Omega$ )

filled with K-gluconate internal solution containing (in mM): $123 \mathrm{~K}$-gluconate, $10 \mathrm{HEPES}, 8 \mathrm{NaCl}, 0.2$

EGTA, $2 \mathrm{MgATP}, 0.3 \mathrm{Na}_{3} \mathrm{GTP}$, and 0.1\% biocytin (pH 7.2 adjusted with $\mathrm{KOH} ; 275 \mathrm{mOsm} / \mathrm{L}$ ). Liquid junction potentials were not corrected during recordings. Input and series resistance were monitored throughout voltage clamp experiments with a $-4 \mathrm{mV}$ step every 30 seconds. Series resistance was required to be $5-30 \mathrm{M} \Omega$ and cells with series resistance changes $>25 \%$ were excluded.

Signals were recorded using a patch clamp amplifier (Axopatch 1D, Molecular Devices, San Jose, CA or IPA, Sutter Instruments, Novato, CA). Signals were filtered at $5 \mathrm{kHz}$ and collected at $20 \mathrm{kHz}$ using IGOR Pro (Wavemetrics) or collected at $10 \mathrm{kHz}$ using SutterPatch software (Sutter Instruments). Light evoked EPSCs and IPSCs were evoked by two blue light pulses (473 nm, 1-10 ms) administered $50 \mathrm{~ms}$ apart, once every $30 \mathrm{~s}$. LHb recordings were generally made in LHb subregions enriched in ChR2-expressing fibers. Recordings were made in voltage-clamp mode, with membrane potential clamped at $V_{\mathrm{m}}=-60 \mathrm{mV}$ and $-40 \mathrm{mV}$, for EPSCs and IPSCs respectively. Light was delivered by an LED coupled to an optic fiber aimed at the recorded cell $(7-10 \mathrm{~mW})$. To calculate connectivity rates, only the first neuron patched per slice was included in order to avoid over sampling from slices or animals with lower infection rates.

Data analysis for electrophysiology. Light pulses were considered to reveal synaptic connections when three conditions were met: (1) the average of 8 traces showed a deviation from baseline $I_{\text {holding }}$ such that the mean trace exceeded $4 \mathrm{SD}$ of $10 \mathrm{~ms}$ baseline period within the $10 \mathrm{~ms}$ window after initiation of light pulse, (2) the putative response was observed in at least 3 independent trials, and (3) the delays from the light stimulation onset of putative responses were time locked ( $<1 \mathrm{~ms}$ jitter) across trials. Latency was calculated as time from start of light pulse to when the rate of rise exceeded $-40,000 \mathrm{~V} / \mathrm{s}$. In some cases DNQX $(10 \mu \mathrm{M})$ or gabazine $(10 \mu \mathrm{M})$ was bath applied to confirm inward and outward currents as AMPA or $\mathrm{GABA}_{\mathrm{A}}$ receptor-mediated, respectively. All measurements of DAMGO effects on EPSCs were 
completed in the presence of gabazine. After recordings, slices were drop fixed in 4\% PFA for at least 2

$494 \mathrm{~h}$ at $4^{\circ} \mathrm{C}$ and processed for biocytin labeling.

495

496

497

498

499

500

501

502

503

504

505

506

507

508

509

510

511

512

513

514

515

516

\section{Combined retrograde tracing and in situ hybridization}

Tracer injections. Male Sprague Dawley rats (300-500 g) were anesthetized with 2-5\% isoflurane. 1\% Fluoro-Gold (FG; FluoroChrome LLC) solution in a $0.1 \mathrm{M}$ cacodylate buffer ( $\mathrm{pH} 7.5$ ) was delivered unilaterally into the $\mathrm{LHb}(-3.4 \mathrm{~mm} \mathrm{AP}, \pm 0.9 \mathrm{~mm} \mathrm{ML}$, and $-5.4 \mathrm{~mm} \mathrm{DV})$ iontophoretically through a stereotaxically positioned glass micropipette (18-25 $\mu \mathrm{m}$ inner diameter) by applying $1 \mu \mathrm{A}, 7 \mathrm{~s}$ pulses at $14 \mathrm{~s}$ intervals for $20 \mathrm{~min}$. The micropipette was then left in place for an additional $10 \mathrm{~min}$ to prevent backflow. Following surgery, rats were singly housed and perfused 3 weeks later.

Tissue Preparation. Rats were anesthetized with chloral hydrate $(0.5 \mathrm{ml} / \mathrm{kg})$ and perfused transcardially with $4 \%(\mathrm{w} / \mathrm{v})$ PFA in $0.1 \mathrm{M}$ phosphate buffer treated with diethylpyrocarbonate (DEPC), $\mathrm{pH}$ 7.3. Brains were post-fixed in $4 \%$ PFA for $2 \mathrm{~h}$ before being transferred to an $18 \%$ sucrose solution $(\mathrm{w} / \mathrm{v}$ in $0.1 \mathrm{M}$ PBS) and stored overnight at $4^{\circ} \mathrm{C}$. Coronal sections of the $\mathrm{LHb}(30 \mu \mathrm{m})$ and LPO $(16 \mu \mathrm{m})$ were prepared.

Phenotyping of retrogradely labeled cells by immunocytochemistry and in situ hybridization. Sections in the $\mathrm{LPO}$ were incubated for $2 \mathrm{~h}$ at $30^{\circ} \mathrm{C}$ with rabbit anti-FG antibody (1:500; AB153; Millipore) supplemented with RNAsin. Sections were then incubated in biotinylated goat anti-rabbit antibody (1:200; BA1000; Vector Laboratories) for $1 \mathrm{~h}$ at $30^{\circ} \mathrm{C}$. Sections were then rinsed and treated with $0.2 \mathrm{~N}$ $\mathrm{HCl}$, rinsed, and then acetylated in $0.25 \%$ acetic anhydride in $0.1 \mathrm{M}$ triethanolamine. Subsequently, sections were rinsed and post-fixed with $4 \%$ PFA, rinsed, and then incubated in a hybridization buffer for $2 \mathrm{~h}$ at $55^{\circ} \mathrm{C}$.

Hybridization was then performed for radioactive detection of MOR mRNA by hybridizing sections for $16 \mathrm{~h}$ at $55^{\circ} \mathrm{C}$ with [35S]- and [33P]-labeled (107 c.p.m./mL) single-stranded antisense probes. Following hybridization, sections were treated with $4 \mu \mathrm{g} / \mathrm{mL}$ of RNAse A at $37^{\circ} \mathrm{C}$ for $1 \mathrm{~h}$, washed with $1 \mathrm{X}$ salinesodium citrate and $50 \%$ formamide for $1 \mathrm{~h}$ at $55^{\circ} \mathrm{C}$, and then with $0.1 \mathrm{X}$ saline-sodium citrate at $68^{\circ} \mathrm{C}$ for 1 
517 h. To visualize FG(+) cells, sections were rinsed with PBS and incubated for $1 \mathrm{~h}$ at $\mathrm{RT}$ in avidin-

518 biotinylated horseradish peroxidase (1:100, ABC kit; Vector Laboratories). Sections were then rinsed, and 519 the peroxidase reaction was developed with $0.05 \%$ 3,3'-diaminobenzidine tetrahydrochloride (DAB) and $520 \quad 0.003 \% \mathrm{H}_{2} \mathrm{O}_{2}$. Sections were then photographed under bright field illumination and mounted on coated 521 slides. Finally, slides were dipped in Ilford K.5 nuclear tract emulsion (Polysciences; 1:1 dilution in 522 double-distilled water) and exposed in the dark at $4{ }^{\circ} \mathrm{C}$ for 3-4 weeks before development and photographs 523 of silver-grain epiluminescence.

524 Data analysis of in situ hybridization studies. Methods for analysis of in situ hybridization material have 525 been described previously ${ }^{46}$. Briefly, pictures were adjusted to match contrast and brightness by using 526 Adobe Photoshop (Adobe Systems). Cell counting was completed independently by three scorers blind to 527 the hypothesis of the study. Radioactive in situ material was analyzed using epiluminescence to increase 528 the contrast of silver grains as described previously ${ }^{59} . \mathrm{FG}(+)$ cells (detected by fluorescence and brown 529 DAB-label) were evaluated for the presence of MOR mRNA: a cell was considered to express MOR 530 mRNA when its soma contained concentric aggregates of silver grains that exceeded background levels.

$531 \quad$ Fiber photometry

532 Surgery. Male and female VGluT2::Cre mice (20-30 g; 6-12 weeks) were anesthetized with 1-5\% 533 isoflurane and secured to a stereotaxic frame. Using a Micro4 controller and UltraMicroPump, $0.2 \mu \mathrm{L}$ of a 534 retrograde, Cre-dependent HSV encoding GCaMP6m (HSV-hEF1 $\alpha$-LS1L-GCaMP6m) was injected into 535 the $\mathrm{LHb}(-1.5 \mathrm{~mm} \mathrm{AP},+0.45 \mathrm{~mm} \mathrm{ML},-3.0 \mathrm{~mm} \mathrm{DV})$. Syringes were left in place for 7-10 min following 536 injections to minimize diffusion. For fiber photometry calcium imaging experiments, a $400 \mu \mathrm{m}$ core optic 537 fiber (Doric Lenses) embedded in a $2.5 \mathrm{~mm}$ ferrule was implanted over the LPO $(+0.5 \mathrm{~mm}$ AP, $+0.8 \mathrm{~mm}$ $538 \mathrm{ML},-5.05 \mathrm{~mm} \mathrm{DV}$ ) and secured to the skull using \#000 screws (Fasteners and Metal products Corp; $539 \quad \# 000-120 \times 1 / 16$ ) and dental cement. Following surgery, mice recovered on a warm heating pad before 
540 being transferred back to the vivarium home cage. Three weeks after the virus and fiber surgery, mice

541 were given either SNI or sham control surgery as described above.

542 Recording. Signals from GCaMP6 were recorded across 10 trials of stimulation in the Hargreaves test

543 using a Plantar Test Instrument. The onset and offset time for each trial was digitized and sent to an

544 RZ5D (Tucker Davis Technologies). For the acquisition of LPO $\rightarrow$ LHB activity, GCaMP6 was excited at

545 two wavelengths (490nm, calcium-dependent signal and $405 \mathrm{~nm}$ isosbestic control) by amplitude

546 modulated signals from two light-emitting diodes reflected off dichroic mirrors and coupled into a $400 \mu \mathrm{m}$

547 0.48NA optic fiber. Signals emitted from GCaMP6m and its isosbestic control channel then returned

548 through the same optic fiber and were acquired using photoreceiver (Doric Lenses), digitized at $1 \mathrm{kHz}$,

549 and then recorded by a real-time signal processor (RZ5D; Tucker Davis Technologies) running the

550 Synapse software suite. Analysis of the resulting signal was then performed using custom-written

551 MATLAB scripts available in a general release form at

552 https://github.com/djamesbarker/FiberPhotometry. Briefly, changes in fluorescence across the

553 experimental session $(\Delta \mathrm{F} / \mathrm{F})$ were calculated by smoothing signals from the isosbestic control channel,

554 scaling the isosbestic control signal by regressing it on the smoothed GCaMP signal, and then generating

555 a predicted $405 \mathrm{~nm}$ signal using the linear model generated during the regression. Calcium independent

556 signals on the predicted $405 \mathrm{~nm}$ channel were then subtracted from the raw GCaMP signal to remove

557 movement, photo-bleaching, and fiber bending artifacts. Signals from the GCaMP channel were then

558 divided by the control signal to generate the $\Delta \mathrm{F} / \mathrm{F}$. Peri-event histograms were then created by averaging

559 changes in fluorescence $(\Delta \mathrm{F} / \mathrm{F})$ across repeated trials during windows encompassing behavioral events of

560 interest. The area under the curve (AUC) was calculated for a pre-stimulation $5 \mathrm{~s}$ baseline commencing -

$56110 \mathrm{~s}$ before paw withdrawal and for the $5 \mathrm{~s}$ period initiated with paw withdrawal. 
563 In rats, unilateral injections of AAV2-hSyn-hChR2(H134R)-mCherry (759 nL) were made throughout 564 anteroposterior range of the ACC ( +2.6 to $-0.4 \mathrm{~mm} \mathrm{AP,}-0.4$ to $-0.5 \mathrm{~mm} \mathrm{ML},-2.6$ to $-2.8 \mathrm{~mm} \mathrm{DV})$. Rats 565 were perfused and brains fixed five weeks later, as described above. Coronal sections $(50 \mu \mathrm{m})$ containing 566 the ACC and the LHb were collected. After verification of the injection site, every sixth slice containing 567 the LHb was rinsed twice with PBS. Tissue was pre-permeabilized in 1:1 EtOH:PBS for 30 min at $4^{\circ} \mathrm{C}$, 568 rinsed briefly in PBS before blocked in $3 \% \mathrm{H}_{2} \mathrm{O}_{2}$ for 10 min. Following PBS washes ( $\left.3 \times 5 \mathrm{~min}\right)$, tissue 569 was blocked in solution containing normal goat serum (NGS, 10\%) for $1 \mathrm{~h}$ at RT. Slices were incubated 570 in rabbit anti-mCherry antibody (1:5000 in PBS + 0.3\% Triton X100 + NGS 10\%; Abcam) overnight at $57144^{\circ} \mathrm{C}$. After PBS washes $(4 \times 10 \mathrm{~min})$, slices were incubated in biotinylated goat anti-rabbit secondary 572 antibody (1:200 in PBS; Vector Laboratories) for $2 \mathrm{~h}$ at $4^{\circ} \mathrm{C}$. Following PBS wash (4x10 min), slices 573 were incubated in VECTASTAIN ${ }^{\circledR}$ ABC Reagent (VECTASTAIN $®$ ABC Kit, Vector Laboratories) for 57430 min followed by peroxidase substrate (DAB Substrate Kit, Vector Laboratories) for 10 min. Once dark 575 brown DAB precipitate formed, slices were rinsed in PBS ( $5 \times 5 \mathrm{~min})$, mounted on glass slides, and cover 576 slipped with DEPEX (Electron Microscopy Sciences). DAB-stained fibers were visualized under 577 brightfield illumination and quantified in Stereo Investigator software (MBF Bioscience) using the virtual 578 isotropic space balls probe ${ }^{60}$.

\section{General experimental design}

580 For behavioral experiments, subject numbers were determined by pilot studies and power analyses (power $581=0.80$, significance level $=0.05$, effect size $=15-30 \%$ ). All behavioral experiments were performed 582 blinded to experimental condition. For immunohistological experiments, three animals with injections 583 targeted at the anterior, middle, and posterior ACC were used to obtain a comprehensive estimation of 584 fibers projecting to the LHb. Electrophysiology experiments were conducted blind to injection site. 
586 Data are expressed as mean \pm SEM or mean with $25^{\text {th }}$ and $75^{\text {th }}$ percentiles as indicated in figure legends 587 and text. Significance was set at $\mathrm{p}<0.05$. Datasets were evaluated to determine whether parametric or 588 non-parametric statistical approaches were most appropriate as indicated in Supplemental Table 1. All 589 tests were two tailed, and statistical analyses were performed in GraphPad Prism or R. The bandwidth for 590 electrophysiology violin plots was determined by Silverman's rule of thumb in Plotly for Python; violin 591 plots for behavior experiments were constructed with heavy kernel density estimations in Prism. Sample 592 sizes are reported in figure panels, legends, and Supplementary Table 1. Outliers, including extreme 593 outliers, are reported in Supplementary Table 1 and were not removed from datasets.

\section{Reporting summary}

596 Further information on research design is available in the Nature Research Reporting Summary linked to 597 this paper.

\section{Data availability}

600 All data described in the main text or extended data are available from the corresponding author upon 601 request.

602

\section{Acknowledgements}

604 The authors would like to thank Ryan Carothers, Gabrielle Mintz, Lucy He, Venkateswaran Ganesh, and 605 Benjamin Snyder for their technical assistance with histology, stereotaxic surgeries, and behavioral 606 studies. This work was supported by National Institutes of Health grants R01DA042025 (to E.B.M.), K08 
NS097632 (to M.W.W.), and the Intramural Research Program (IRP) of the National Institute on Drug Abuse (IRP/NIDA/NIH) and a NIDA K99/R00 pathway to independence award (DA043572) to D.J.B.

609

\section{Competing Interests Statement}

611 The authors have no competing interests.

612

613

\section{Figure Legends}

Figure 1. MOR activation in the LHb relieves allodynia and generates negative reinforcement in rats

615 with neuropathic pain. a, Timeline of behavioral experiments. Lower inset, example injection site

616 centered in LHb. Scale bar $=500 \mu \mathrm{m}$. b, (Left) Violin plots of 50\% mechanical withdrawal thresholds

617 following saline or $10 \mu \mathrm{M}$ DAMGO microinjections into the LHb in sham males $(\mathrm{n}=9)$, SNI males $(\mathrm{n}=$

618 8), and SNI females $(\mathrm{n}=9)$. Sham males: Wilcoxon signed rank test, $\mathrm{V}=1.5, \mathrm{p}=1$. SNI males: Wilcoxon

619 signed rank test, $\mathrm{V}=2, \mathrm{p}=0.050$. Females trended towards an increase in threshold following DAMGO

620 infusion: Wilcoxon signed rank test, $\mathrm{V}=7, \mathrm{p}=0.074$. (Right) Mechanical withdrawal thresholds in male

621 rats with i.c.v. cannulae, with SNI $(n=12)$ or sham $(n=9)$ injuries. Sham: Wilcoxon signed rank test, V

$622=7, p=0.53$. SNI: Wilcoxon signed rank test, $\mathrm{V}=31, \mathrm{p}=0.080$. $\mathbf{c}$, Difference scores before and after

623 conditioning with intracranial microinjections. (Left) Males with SNI developed a preference for the

624 chamber paired with intra-LHb DAMGO. Two-way mixed ANOVA, significant interaction between

$625 \mathrm{SNI} /$ sham and baseline/test $\mathrm{F}(1,13)=15.93, \mathrm{p}=0.002$; post hoc effect group on test day adjusted $\mathrm{p}=$

$6260.01 ;$ paired t-tests, sham adjusted $\mathrm{p}=1 ; \mathrm{SNI}$ adjusted $\mathrm{p}=0.016$. Female SNI animals trended towards a

627 preference for the DAMGO-paired chamber: Paired t-test $\mathrm{p}=0.054$. (Right) i.c.v. DAMGO did not

628 produce a preference in sham or SNI male rats: Two-way mixed ANOVA, $F(1,19)=2.239, p=0.15$. d,

629 Mechanical withdrawal thresholds in sham $(\mathrm{n}=6)$ and SNI $(\mathrm{n}=9)$ female rats following $100 \mu \mathrm{M}$

630 DAMGO infusions into the LHb. Sham: Wilcoxon signed rank test, $\mathrm{V}=5, \mathrm{p}=1$. SNI: Wilcoxon signed 
rank test, $\mathrm{V}=6, \mathrm{p}=0.40$. e, Place conditioning of sham and SNI females to intra-LHb $100 \mu \mathrm{M}$ DAMGO vs saline. Females with SNI developed a significant CPP: Two-way mixed ANOVA, significant interaction between $\mathrm{SNI} /$ sham and baseline/test $\mathrm{F}(1,13)=6.234, \mathrm{p}=0.027$; post hoc effect group on test day adjusted $\mathrm{p}=0.07$; paired t-tests, sham adjusted $\mathrm{p}=0.48 ;$ SNI adjusted $\mathrm{p}=0.035$. Means represented by horizontal lines; SEM and quartiles indicated by vertical lines with and without caps, respectively. *p $\leq 0.05, * * \mathrm{p}<0.01$

Figure 2. Ongoing pain does not alter MOR agonist synaptic effects or probability of glutamate release in the $\mathrm{LHb}$. Responses to the MOR agonist DAMGO were recorded in voltage clamp, $\mathrm{V}_{\mathrm{m}}=-60 \mathrm{mV}$. a, In animals with SNI, a subset of neurons responded to bath application of the MOR agonist DAMGO (500 $\mathrm{nM}$ ) with a time locked outward current that was reversed with the MOR selective antagonist CTAP (500 $\mathrm{nM})$. b, Postsynaptic holding current changes in response to bath application of DAMGO were not different between neurons from naïve and SNI rats: Unpaired t-test $\mathrm{df}=32, \mathrm{t}=0.892, \mathrm{p}=0.379$. $\mathbf{c}$, In rats with SNI, DAMGO (500 nM) induced a time locked inhibition of electrically evoked glutamatergic EPSCs, and CTAP (500 nM) reversed the effect. d, This DAMGO inhibition of electrically evoked EPSC amplitude was not different between LHb neurons from naïve and SNI rats: Unpaired t-test $\mathrm{df}=21, \mathrm{t}=$ $0.137, \mathrm{p}=0.89$. Baseline probability of release at glutamatergic synapses was similar between LHb neurons in naïve and SNI rats as measured by (e) paired pulse ratio (P2/P1) of electrically stimulated EPSCs (50 ms inter stimulus interval): Unpaired t-test, $\mathrm{df}=24, \mathrm{t}=0.102, \mathrm{p}=0.92 ;(\mathbf{f})$ baseline spontaneous EPSC frequency: Unpaired t-test unequal variances, $\mathrm{df}=37, \mathrm{t}=-0.17, \mathrm{p}=0.87$; and $(\mathbf{g})$ spontaneous EPSC amplitude: Unpaired t-test unequal variances, $\mathrm{df}=38, \mathrm{t}=-0.09, \mathrm{p}=0.93$. Data from naïve rats previously published in ${ }^{16}$. 
Figure 3. Glutamatergic inputs to the LHb from the LPO are inhibited by MOR activation. a, Schematic diagram of AAV2-hSyn-hChR2(H134R)-mCherry injection targets which project to the LHb. $\mathbf{b}$, Proportions of $\mathrm{LHb}$ neurons receiving functional synaptic connections from six input regions measured with optogenetic stimulation of terminals expressing ChR2. c, Among connected neurons, venn diagrams depict the numbers of LHb cells receiving optically stimulated glutamatergic and/or GABAergic synaptic input. d, (Left) Experiment design: DAMGO (500 nM) was applied during optical synaptic stimulation experiments to probe for functional MOR presynaptic control of glutamate release from specific inputs. (Right) Change from baseline amplitude of optically-evoked EPSCs during DAMGO application. EPSCs from LPO terminal stimulation were most strongly inhibited by MOR activation: One-way ANOVA, $\mathrm{df}=$ 4, $\mathrm{F}=4.11, \mathrm{p}=0.0057$ followed by Tukey HSD pairwise comparisons. $\mathbf{e}$, (Left) Representative traces of optically-evoked EPSCs in an LHb neuron from a rat with ChR2 expressed in inputs from the LPO. DAMGO decreased the amplitude of light-evoked EPSC, which was partially reversed by the selective MOR antagonist CTAP $(500 \mathrm{nM})$. Rise time was $<2 \mathrm{~ms}$, indicative of a monosynaptic response. (Right) Summary time course of the DAMGO inhibition across all optically-evoked LPO-LHb EPSCs $(\mathrm{n}=12)$. f, LPO inputs to the LHb in female rats (left) had a similar overall connectivity rate but (middle) more GABAergic connections than glutamate connections. (Right) DAMGO inhibited light-evoked glutamatergic synaptic inputs from the LPO to the $\mathrm{LHb}$ in female rats as well.

Figure 4. LPO inputs to the LHb highly express MOR $m R N A$. a, Schematic of unilateral iontophoretic deposition of the retrograde tracer FG into the LHb. b, FG injection sites in LHb ( $\mathrm{n}=3$ rats). c, (Left and middle) Representative brightfield images of FG-positive/MOR-positive somas (yellow arrowhead) and FG-positive/MOR-negative somas (blue arrowhead) in the LPO. Scale bar $=20 \mu \mathrm{m}$. (Right) Darkfield image of in situ hybridization localization for MOR mRNA (OPRM1). d, Number of MOR-positive/FGpositive (yellow) and MOR-negative/FG-positive (blue) cells in various basal forebrain regions. e, Donut plot depicting proportions of total MOR-positive inputs to the LHb from various basal forebrain regions. 
f, Locations of FG-positive/MOR-positive (red) and FG-positive/MOR-negative (blue) somata throughout basal forebrain. Co-labeled cells are separated from the singly labelled population for display purposes.

Figure 5. $L H b$ MOR activation relieves aversiveness of glutamatergic LPO input to the $L H b$. a, (Top) Schematic diagram of HSV-hEF1 $\alpha$-LS1L-GCaMP6m injection into the LHb of VGluT2::Cre mice, to induce expression of GCaMP6m in glutamatergic LPO neurons innervating the LHb, and optic fiber implant for fiber photometry imaging in the LHb-projecting LPO neurons. (Bottom) Experimental timeline. $\mathbf{b}$, Mice with SNI (green, $\mathrm{n}=5$ ) exhibited significantly larger changes in GCaMP6m fluorescence time-locked to paw withdrawal in response to thermal stimulation in the Hargreaves apparatus compared to sham controls (grey, $\mathrm{n}=9$ ): Two-way ANOVA, $\mathrm{F}(1,12)=5.439, \mathrm{p}=0.038$; Holm-Sidak post-hoc test, $\mathrm{p}=0.0074$. $\mathbf{c}$, (Left) Schematic diagram of dual virus injections to express ChR2-mCherry or mCherry selectively in LPO neurons that project to the LHb, optic fiber implantation targeting the LPO cell bodies, and cannula implantation targeting the LHb for experiments in male rats. (Right) Rats received bilateral blue light stimulation of the LPO during all conditioning sessions and in both chambers, sides paired with intra-LHb saline or DAMGO. d, Representative images of Credependent ChR2-mCherry fluorescence in LPO cell bodies. (Left) Scale bar $=250 \mu \mathrm{m}$. (Right) Lesion from optic fiber (“o.f.") implant amidst ChR2-mCherry-expressing cell bodies. Scale bar $=50 \mu \mathrm{m} . \mathbf{e}$, Only animals with active ChR2 $(n=8)$, but not mCherry controls $(n=8)$, developed a preference for the intra-LHb DAMGO paired side of the place conditioning apparatus. Two-way mixed ANOVA, significant interaction between ChR2-mCherry/mCherry and baseline/test $F(1,14)=9.982, p=0.007$; post hoc effect group on test day adjusted $p=0.006$; paired t-tests, mCherry adjusted $p=0.427$; ChR2mCherry adjusted $\mathrm{p}=0.0027 .{ }^{* *} \mathrm{p}<0.01, * * * \mathrm{p}<0.005$

\section{Extended Data Figure Legends}


Extended Data Figure 1. Further characterization of neuropathic pain and inflammatory pain models

704 and the impact of intra-LHb MOR activation in rat. a, (Left) Violin plots of withdrawal latency to heat in 705 sham $(\mathrm{n}=9)$, male $(\mathrm{n}=8)$ and female $(\mathrm{n}=9)$ rats with SNI after saline vs DAMGO $(10 \mu \mathrm{M})$

706 microinjections into the LHb. Sham males: Paired t-test, $\mathrm{df}=8, \mathrm{t}=1.06, \mathrm{p}=0.32$; SNI males: Paired t707 test, $\mathrm{df}=7, \mathrm{t}=-1.92, \mathrm{p}=0.096$; SNI females: Paired t-test, $\mathrm{df}=8, \mathrm{t}=-1.4, \mathrm{p}=0.199$. (Right) i.c.v. sham

708

709

710

711

712

713

714

715

716

717

718

719

720

721

male: Paired t-test, $\mathrm{df}=8, \mathrm{t}=-0.08, \mathrm{p}=0.938$. i.c.v. SNI male: Paired t-test, $\mathrm{df}=11, \mathrm{t}=3.2, \mathrm{p}=0.00846$.

To test if the effects of MOR activation in the LHb generalize to other forms of pain, we repeated the manipulations above with an inflammatory model of pain. Male rats received an intradermal injection of CFA or sterile saline into the plantar aspect of the hindpaw, $\mathbf{b}$, Schematic diagram of inflammatory injury preparations and cannulation targeting the LHb. c, Mechanical withdrawal thresholds in sham and CFAinjured rats. In animals with CFA, we observed an increase in the average withdrawal threshold following intra-LHb DAMGO $(10 \mu \mathrm{M})$ compared to saline, indicating a reduction in mechanical allodynia: Paired ttest, $\mathrm{df}=8, \mathrm{t}=-2.89, \mathrm{p}=0.0202$; but not shams Wilcoxon signed rank test, $\mathrm{V}=1.5, \mathrm{p}=1$. $\mathbf{d}$, Withdrawal latency to heat. Similar to results in rats with SNI, DAMGO in the LHb did not reverse the CFA-induced decrease in withdrawal latency to heat. Sham: Paired t-test, $\mathrm{df}=8, \mathrm{t}=1.06, \mathrm{p}=0.32$. CFA: Wilcoxon signed rank test, $\mathrm{V}=12, \mathrm{p}=0.25$. e, Rats with CFA injury also did not develop a preference for the DAMGO-paired chamber in the place conditioning paradigm: Two-way mixed ANOVA, significant interaction between CFA/sham and baseline/test $\mathrm{F}(1,14)=0.474, \mathrm{p}=0.502$; paired t-tests, sham adjusted $\mathrm{p}=0.286 ;$ SNI adjusted $\mathrm{p}=0.761$. The discrepancy between place conditioning results of our neuropathic and inflammatory pain animals may be due to the natural history of the injury caused in the CFA model: whereas SNI animals underwent permanent nerve ligation, animals injected with CFA presented with transient swelling and erythema of the hindpaw. Within a few days of CFA injection, this phenotype had resolved in our rats. f, Mechanical allodynia also reversed quickly: in a separate cohort of rats, all subjects displayed a sharp decline in withdrawal threshold from baseline on day 1 following CFA injection but the injury-induced allodynia returned to control levels by day 5 in many animals. Individual animals were 
tested daily; each line represents a male rat. g, Average across rats shows that our CFA-induced reduction in mechanical allodynia reversed within a few days. Since our place conditioning training exceeds this time window, resolution of the injury probably occurred before training was completed, thus preventing the formation of a robust association between pain relief and place conditioning apparatus context to produce a CPP. ${ }^{*} \mathrm{p} \leq 0.05,{ }^{* *} \mathrm{p} \leq 0.01$

Extended Data Figure 2. Neurons receiving functional synaptic inputs from the five brain regions showing connectivity were distributed throughout the LHb. Locations of neurons found connected using whole-cell recordings from optogenetic stimulation of terminals arising from each input tested. Locations are based on biocytin immunohistochemistry and low magnification images taken on the recording microscope where the recorded cell is centered within the field of view. Color indicates source of innervation.

Extended Data Figure 3. ACC minimally innervates the $L H b$. a, Diagram of the extent of unilateral AAV2-hSyn-hChR2(H134R)-mCherry injection sites ( $\mathrm{n}=9$ male rats) throughout anteroposterior range of the ACC for anterograde tracing study. b, Example ipsilateral DAB-positive fibers (black) visualized under brightfield illumination. Fibers heavily innervate the MDL, which abuts the lateral edge of the LHb, while sparse to no fibers innervate the LHb. Contralateral innervation of LHb and MDL was negligible compared to the ipsilateral side, and therefore were omitted from our analysis. (Left) Scale bar $=250 \mu \mathrm{m}$. (Right) Scale bar $=50 \mu \mathrm{m}$. c, Average stereologically-quantified DAB-positive fiber lengths throughout anteroposterior range of the $\mathrm{LHb}$, compared to the MDL innervation in the same coronal slice. MannWhitney test, two-tailed: $\mathrm{U}=0, \mathrm{p}=0.0006$. 
Extended Data Figure 4. Sources of glutamatergic inputs to the rat LHb vary in strength and delay in

752 synaptic transmission. a, We observed some variations between inputs in the mean light evoked EPSCs

$753\left(\mathrm{~V}_{\mathrm{m}}=-60 \mathrm{mV}\right)$. In particular, excitatory inputs from the VTA were consistently small: Kruskal-Wallis $\chi^{2}$

$754=25.5, \mathrm{df}=4, \mathrm{p}=0.00004$, followed by Dunn Test for pairwise comparisons. $\mathbf{b}$, Differences were also

755 detected in the delay to the onset of light evoked EPSCs to LHb neurons from these different sources,

756 with inputs from LH showing the fastest response. Although these data deviate from a normal

757 distribution (Shapiro's test, $\mathrm{p}=0.0000001$ ), KDEs (violins) are consistent with continuous distributions,

758 suggesting reliable polysynaptic events were rarely detected. c, We observed some variations between

759 inputs in the mean light evoked IPSCs $\left(\mathrm{V}_{\mathrm{m}}=-40 \mathrm{mV}\right)$. While there were no statistically significant

760 differences in amplitudes detected, the mean inhibitory input from the VP was particularly small:

761 Kruskal-Wallis $\chi 2=2.3, \mathrm{df}=4, \mathrm{p}=0.7 . * * \mathrm{p}<0.01, * * * \mathrm{p}<0.005, * * * * \mathrm{p}<0.0005$

Extended Data Figure 5. Optogenetic experiments detect local glutamate, but not GABA, functional connections in rat LHb. AAV2-hSyn-hChR2(H134R)-mCherry was stereotaxically injected into the LHb at least 4 weeks prior to ex vivo whole cell recordings in the LHb to detect local synaptic connections. Neurons were recorded blind to ChR2 expression, therefore in some cases the patched neuron expressed ChR2. Therefore, in addition to connectivity criteria used for other afferent inputs, in these experiments only light evoked inward currents that were blocked by $10 \mu \mathrm{M}$ DNQX were considered glutamatergic connections, and direct ChR2 induced inward currents were subtracted out for the quantification illustrated here. Each cell was probed for both glutamate and GABA inputs in voltage clamp by holding neurons at $\mathrm{V}_{\mathrm{m}}=-60 \mathrm{mV}$ and $-40 \mathrm{mV}$, light pulse durations 1,5 , and $10 \mathrm{~ms}$ durations. a, Example recording at $\mathrm{V}_{\mathrm{m}}=-60 \mathrm{mV}$ showing a light evoked response that was blocked by DNQX. b, Example recording at $\mathrm{V}_{\mathrm{m}}=-40 \mathrm{mV}$ with minimal outward current response within $7 \mathrm{~ms}$ of light pulse. c, Summary

774 of all LHb recordings tested in this experiment. Filled circles represent cells where responses could be 775 classified as local ChR2 induced synaptic transmission. When no clear response was detected, the 
measure indicated is the difference between the mean $I_{\text {holding }}$ of the baseline $100 \mathrm{~ms}$ period just prior to the light pulse and the mean $I_{\text {holding }} 2 \mathrm{~ms}$ period starting $2 \mathrm{~ms}$ after initiation of the light pulse, consistent with monosynaptic delay timing. None of the recordings made at $\mathrm{V}_{\mathrm{m}}=-40 \mathrm{mV}$ met the criteria for a response to light stimulation with an outward current.

\section{Extended Data Figure 6. Isolated monosynaptic glutamatergic inputs from LPO to LHb neurons are} inhibited by MOR activation. a, Example light evoked EPSC responses in an LHb neuron from a rat with ChR2 expression in LPO neurons. This response persisted in monosynaptic isolation by $500 \mathrm{nM}$ TTX and $100 \mu \mathrm{M} 4 \mathrm{AP}$ (green), and this isolated response was inhibited by $500 \mathrm{nM}$ DAMGO (magenta). b, Summary of DAMGO effects on isolated monosynaptic EPSC inputs to LHb neurons expressed as \% of baseline monosynaptic response (left) and as raw EPSC magnitudes (right). Paired t-test, $\mathrm{df}=4, \mathrm{t}=-5.1$, $\mathrm{p}=0.007 . * * \mathrm{p}<0.01$

\section{Extended Data Figure 7. Mice with SNI show hypersensitivity to heat and increased activity in LHb-} projecting LPO neurons during paw withdrawal from thermal stimulation. a, Mice with SNI show a reduced latency to withdraw their paw following thermal stimulation in the Hargreaves task. Unpaired ttest, $\mathrm{t}(12)=3.007, \mathrm{p}=0.011 . \mathbf{b}$, VGluT2-expressing LPO neurons that project to the LHb expressed GCaMP6m and showed a greater calcium response during paw withdrawal to Hargreaves thermal stimulation (area under the curve, deviation from baseline fluorescence) in SNI animals $(n=5)$ compared to sham controls $(n=9)$ : Two-way ANOVA, $F(1,12)=5.4, p=0.038$; Holm-Sidak post-hoc test, $\mathrm{p}=$ 0.0074. $* * \mathrm{p}<0.01$

\section{Extended Data Figure 8. Locations and spread of bilateral ChR2 injections to the LPO in rats used for} in vivo optogenetic experiments. 


\section{References}

1. Matsumoto, M. \& Hikosaka, O. Representation of negative motivational value in the primate lateral habenula. Nat Neurosci 12, 77-84 (2009).

2. Benabid, A. L. \& Jeaugey, L. Cells of the rat lateral habenula respond to high-threshold somatosensory inputs. Neurosci Lett 96, 289-94 (1989).

3. Dafny, N. \& Qiao, J. T. Habenular neuron responses to noxious input are modified by dorsal raphe stimulation. Neurol. Res. 12, 117-121 (1990).

4. Nagao, M., Kamo, H., Akiguchi, I. \& Kimura, J. Induction of c-Fos-like protein in the lateral habenular nucleus by persistent noxious peripheral stimulation. Neurosci. Lett. 151, 37-40 (1993).

5. Huang, T. et al. Identifying the pathways required for coping behaviours associated with sustained pain. Nature 565, 86-90 (2019).

6. Matsumoto, M. \& Hikosaka, O. Lateral habenula as a source of negative reward signals in dopamine neurons. Nature 447, 1111-1115 (2007).

7. Li, B. et al. Synaptic potentiation onto habenula neurons in the learned helplessness model of depression. Nature 470, 535-539 (2011).

8. Poller, W. C., Madai, V. I., Bernard, R., Laube, G. \& Veh, R. W. A glutamatergic projection from the lateral hypothalamus targets VTA-projecting neurons in the lateral habenula of the rat. Brain Res 1507, 45-60 (2013).

9. Herkenham, M. \& Nauta, W. J. Afferent connections of the habenular nuclei in the rat. A horseradish peroxidase study, with a note on the fiber-of-passage problem. J Comp Neurol 173, 123-46 (1977).

10. Vertes, R. P. Analysis of projections from the medial prefrontal cortex to the thalamus in the rat, with emphasis on nucleus reuniens. J. Comp. Neurol. 442, 163-187 (2002).

11. Yetnikoff, L., Cheng, A. Y., Lavezzi, H. N., Parsley, K. P. \& Zahm, D. S. Sources of input to the rostromedial tegmental nucleus, ventral tegmental area, and lateral habenula compared: A study in rat. J Comp Neurol 523, 2426-56 (2015). 
12. Kim, U. \& Lee, T. Topography of descending projections from anterior insular and medial prefrontal regions to the lateral habenula of the epithalamus in the rat. Eur J Neurosci 35, 1253-69 (2012).

13. Araki, M., McGeer, P. L. \& Kimura, H. The efferent projections of the rat lateral habenular nucleus revealed by the PHA-L anterograde tracing method. Brain Res. 441, 319-330 (1988).

14. Quina, L. A. et al. Efferent pathways of the mouse lateral habenula. J Comp Neurol 523, 32-60 (2015).

15. Cohen, S. R. \& Melzack, R. Morphine injected into the habenula and dorsal posteromedial thalamus produces analgesia in the formalin test. Brain Res. 359, 131-139 (1985).

16. Margolis, E. B. \& Fields, H. L. Mu Opioid Receptor Actions in the Lateral Habenula. PLoS One 11, e0159097 (2016).

17. Decosterd, I. \& Woolf, C. J. Spared nerve injury: an animal model of persistent peripheral neuropathic pain. PAIN 87, 149-158 (2000).

18. Miaskowski, C. et al. Does opioid analgesia show a gender preference for females? Pain Forum $\mathbf{8}$, 34-44 (1999).

19. Kest, B., Sarton, E. \& Dahan, A. Gender differences in opioid-mediated analgesia: animal and human studies. Anesthesiology 93, 539-547 (2000).

20. Kepler, K. L. et al. Gender effects and central opioid analgesia. Pain 45, 87-94 (1991).

21. Thompson, S. J. et al. Chronic neuropathic pain reduces opioid receptor availability with associated anhedonia in rat. Pain 159, 1856-1866 (2018).

22. Porreca, F. et al. Spinal opioid mu receptor expression in lumbar spinal cord of rats following nerve injury. Brain Res. 795, 197-203 (1998).

23. Zhang, X. et al. Down-regulation of mu-opioid receptors in rat and monkey dorsal root ganglion neurons and spinal cord after peripheral axotomy. Neuroscience 82, 223-240 (1998). 
24. Shabel, S. J., Proulx, C. D., Trias, A., Murphy, R. T. \& Malinow, R. Input to the lateral habenula from the basal ganglia is excitatory, aversive, and suppressed by serotonin. Neuron 74, 475-481 (2012).

25. Lecca, S. et al. Aversive stimuli drive hypothalamus-to-habenula excitation to promote escape behavior. eLife 6, (2017).

26. Hu, H., Cui, Y. \& Yang, Y. Circuits and functions of the lateral habenula in health and in disease. Nat. Rev. Neurosci. 21, 277-295 (2020).

27. Root, D. H., Mejias-Aponte, C. A., Qi, J. \& Morales, M. Role of glutamatergic projections from ventral tegmental area to lateral habenula in aversive conditioning. J Neurosci 34, 13906-10 (2014).

28. Barker, D. J. et al. Lateral Preoptic Control of the Lateral Habenula through Convergent Glutamate and GABA Transmission. Cell Rep. 21, 1757-1769 (2017).

29. Fields, H. State-dependent opioid control of pain. Nat Rev Neurosci 5, 565-75 (2004).

30. Tooley, J. et al. Glutamatergic Ventral Pallidal Neurons Modulate Activity of the HabenulaTegmental Circuitry and Constrain Reward Seeking. Biol. Psychiatry 83, 1012-1023 (2018).

31. Johansen, J. P., Fields, H. L. \& Manning, B. H. The affective component of pain in rodents: direct evidence for a contribution of the anterior cingulate cortex. Proc. Natl. Acad. Sci. U. S. A. 98, 8077$8082(2001)$.

32. LaGraize, S. C., Borzan, J., Peng, Y. B. \& Fuchs, P. N. Selective regulation of pain affect following activation of the opioid anterior cingulate cortex system. Exp. Neurol. 197, 22-30 (2006).

33. Kim, U. \& Chang, S. Y. Dendritic morphology, local circuitry, and intrinsic electrophysiology of neurons in the rat medial and lateral habenular nuclei of the epithalamus. $J$ Comp Neurol 483, 236-50 (2005).

34. Flanigan, M. E. et al. Orexin signaling in GABAergic lateral habenula neurons modulates aggressive behavior in male mice. Nat. Neurosci. 23, 638-650 (2020). 
872

35. Zhang, L. et al. A GABAergic cell type in the lateral habenula links hypothalamic homeostatic and midbrain motivation circuits with sex steroid signaling. Transl. Psychiatry 8, 50 (2018).

36. Quina, L. A., Walker, A., Morton, G., Han, V. \& Turner, E. E. GAD2 Expression Defines a Class of Excitatory Lateral Habenula Neurons in Mice that Project to the Raphe and Pontine Tegmentum. eNeuro 7, (2020).

37. Wallace, M. L. et al. Anatomical and single-cell transcriptional profiling of the murine habenular complex. eLife 9, (2020).

38. Mansour, A., Khachaturian, H., Lewis, M. E., Akil, H. \& Watson, S. J. Autoradiographic differentiation of mu, delta, and kappa opioid receptors in the rat forebrain and midbrain. J. Neurosci. Off. J. Soc. Neurosci. 7, 2445-2464 (1987).

39. Kang, S. et al. Downregulation of M-channels in lateral habenula mediates hyperalgesia during alcohol withdrawal in rats. Sci. Rep. 9, 2714 (2019).

40. Li, J., Li, Y., Zhang, B., Shen, X. \& Zhao, H. Why depression and pain often coexist and mutually reinforce: Role of the lateral habenula. Exp. Neurol. 284, 106-113 (2016).

41. Li, Y. et al. Role of the Lateral Habenula in Pain-Associated Depression. Front. Behav. Neurosci. 11, 31 (2017).

42. Weiss, T. \& Veh, R. W. Morphological and electrophysiological characteristics of neurons within identified subnuclei of the lateral habenula in rat brain slices. Neuroscience 172, 74-93 (2011).

43. Metzger, M. et al. Habenular connections with the dopaminergic and serotonergic system and their role in stress-related psychiatric disorders. Eur. J. Neurosci. (2019) doi:10.1111/ejn.14647.

44. Meye, F. J. et al. Shifted pallidal co-release of GABA and glutamate in habenula drives cocaine withdrawal and relapse. Nat. Neurosci. 19, 1019-1024 (2016).

45. Shabel, S. J., Proulx, C. D., Piriz, J. \& Malinow, R. Mood regulation. GABA/glutamate co-release controls habenula output and is modified by antidepressant treatment. Science 345, 1494-8 (2014). 
46. Root, D. H. et al. Selective Brain Distribution and Distinctive Synaptic Architecture of Dual Glutamatergic-GABAergic Neurons. Cell Rep. 23, 3465-3479 (2018).

47. Newman, H. M., Stevens, R. T. \& Apkarian, A. V. Direct spinal projections to limbic and striatal areas: anterograde transport studies from the upper cervical spinal cord and the cervical enlargement in squirrel monkey and rat. J. Comp. Neurol. 365, 640-658 (1996).

48. Burstein, R., Cliffer, K. D. \& Giesler, G. J. Direct somatosensory projections from the spinal cord to the hypothalamus and telencephalon. J. Neurosci. Off. J. Soc. Neurosci. 7, 4159-4164 (1987).

49. Hori, T., Oka, T., Hosoi, M. \& Aou, S. Pain modulatory actions of cytokines and prostaglandin E2 in the brain. Ann. N. Y. Acad. Sci. 840, 269-281 (1998).

50. Ono, T. \& Nakamura, K. Learning and integration of rewarding and aversive stimuli in the rat lateral hypothalamus. Brain Res. 346, 368-373 (1985).

51. Almli, C. R. \& McMullen, N. T. Ontogeny of lateral preoptic unit activity in rats. Brain Res. Bull. 4, 773-781 (1979).

52. Vowles, K. E. et al. Rates of opioid misuse, abuse, and addiction in chronic pain: a systematic review and data synthesis. Pain 156, 569-576 (2015).

53. Zadina, J. E. et al. Endomorphin analog analgesics with reduced abuse liability, respiratory depression, motor impairment, tolerance, and glial activation relative to morphine. Neuropharmacology 105, 215-227 (2016).

54. Ehrlich, A. T. et al. Expression map of 78 brain-expressed mouse orphan GPCRs provides a translational resource for neuropsychiatric research. Commun. Biol. 1, 102 (2018).

55. Wagner, F., Bernard, R., Derst, C., French, L. \& Veh, R. W. Microarray analysis of transcripts with elevated expressions in the rat medial or lateral habenula suggest fast GABAergic excitation in the medial habenula and habenular involvement in the regulation of feeding and energy balance. Brain Struct. Funct. 221, 4663-4689 (2016). 
920 56. Broms, J. et al. Monosynaptic retrograde tracing of neurons expressing the G-protein coupled

921 receptor Gpr151 in the mouse brain. J. Comp. Neurol. 525, 3227-3250 (2017).

922 57. Paxinos, G. \& Watson, C. The Rat Brain in Stereotaxic Coordinates, Compact. (Academic Press, 923 1997).

924 58. Chaplan, S. R., Bach, F. W., Pogrel, J. W., Chung, J. M. \& Yaksh, T. L. Quantitative assessment of 925 tactile allodynia in the rat paw. J. Neurosci. Methods 53, 55-63 (1994).

926 59. Yamaguchi, T., Wang, H.-L., Li, X., Ng, T. H. \& Morales, M. Mesocorticolimbic glutamatergic

927 pathway. J. Neurosci. Off. J. Soc. Neurosci. 31, 8476-8490 (2011).

928

60. Mouton, P. R., Gokhale, A. M., Ward, N. L. \& West, M. J. Stereological length estimation using 929 spherical probes. J. Microsc. 206, 54-64 (2002).

930 


\section{Extended Data Figure 1}
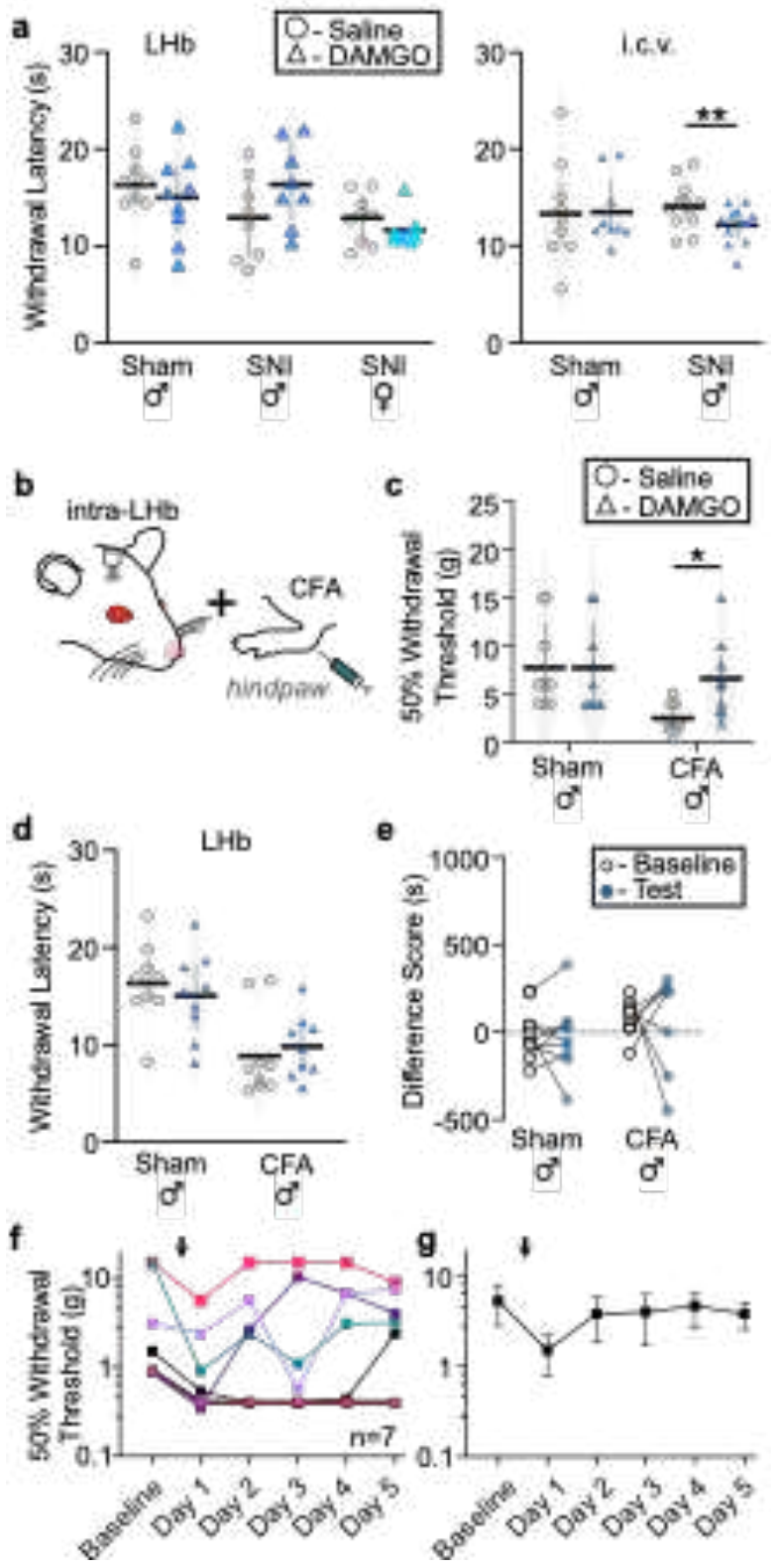

Extended Data Figure 1. Further characterization of neuropathic pain and inflammatory pain models and the impact of intra- $\mathrm{LHb}$ $M O R$ activation in rat. a, (Left) Violin plots of withdrawal latency to heat in sham $(n=9)$, male $(n=8)$ and female $(n=9)$ rats with SNI after saline vs DAMGO $(10 \mu \mathrm{M})$ microinjections into the LHb. Sham males: Paired t-test, $\mathrm{df}=8, \mathrm{t}=1.06, \mathrm{p}=0.32$; SNI males: Paired ttest, $\mathrm{df}=7, \mathrm{t}=-1.92, \mathrm{p}=0.096$; SNI females: Paired t-test, $\mathrm{df}=8, \mathrm{t}=$ -1.4, $\mathrm{p}=0.199$. (Right) i.c.v. sham male: Paired t-test, $\mathrm{df}=8, \mathrm{t}=$ $0.08, \mathrm{p}=0.938$. i.c.v. SNI male: Paired t-test, $\mathrm{df}=11, \mathrm{t}=3.2, \mathrm{p}=$ 0.00846 . To test if the effects of MOR activation in the LHb generalize to other forms of pain, we repeated the manipulations above with an inflammatory model of pain. Male rats received an intradermal injection of CFA or sterile saline into the plantar aspect of the hindpaw, $\mathbf{b}$, Schematic diagram of inflammatory injury preparations and cannulation targeting the LHb. c, Mechanical withdrawal thresholds in sham and CFA-injured rats. In animals with CFA, we observed an increase in the average withdrawal threshold following intra-LHb DAMGO $(10 \mu \mathrm{M})$ compared to saline, indicating a reduction in mechanical allodynia: Paired t-test, $\mathrm{df}=8, \mathrm{t}$ $=-2.89, \mathrm{p}=0.0202$; but not shams Wilcoxon signed rank test, $\mathrm{V}=$ $1.5, \mathrm{p}=1$. d, Withdrawal latency to heat. Similar to results in rats with SNI, DAMGO in the LHb did not reverse the CFA-induced decrease in withdrawal latency to heat. Sham: Paired t-test, $\mathrm{df}=8, \mathrm{t}=$ 1.06, $\mathrm{p}=0.32$. CFA: Wilcoxon signed rank test, $\mathrm{V}=12, \mathrm{p}=0.25$. e, Rats with CFA injury also did not develop a preference for the DAMGO-paired chamber in the place conditioning paradigm: Twoway mixed ANOVA, significant interaction between CFA/sham and baseline/test $\mathrm{F}(1,14)=0.474, \mathrm{p}=0.502$; paired t-tests, sham adjusted $\mathrm{p}=0.286$; SNI adjusted $\mathrm{p}=0.761$. The discrepancy between place conditioning results of our neuropathic and inflammatory pain animals may be due to the natural history of the injury caused in the CFA model: whereas SNI animals underwent permanent nerve ligation, animals injected with CFA presented with transient swelling and erythema of the hindpaw. Within a few days of CFA injection, this phenotype had resolved in our rats. f, Mechanical allodynia also reversed quickly: in a separate cohort of rats, all subjects displayed a sharp decline in withdrawal threshold from baseline on day 1 following CFA injection but the injury-induced allodynia returned to control levels by day 5 in many animals. Individual animals were tested daily; each line represents a male rat. g, Average across rats shows that our CFA-induced reduction in mechanical allodynia reversed within a few days. Since our place conditioning training exceeds this time window, resolution of the injury probably occurred before training was completed, thus preventing the formation of a robust association between pain relief and place conditioning apparatus context to produce a CPP. ${ }^{*} \mathrm{p} \leq 0.05,{ }^{*} \mathrm{p} \leq$ 0.01 


\section{Extended Data Figure 2}

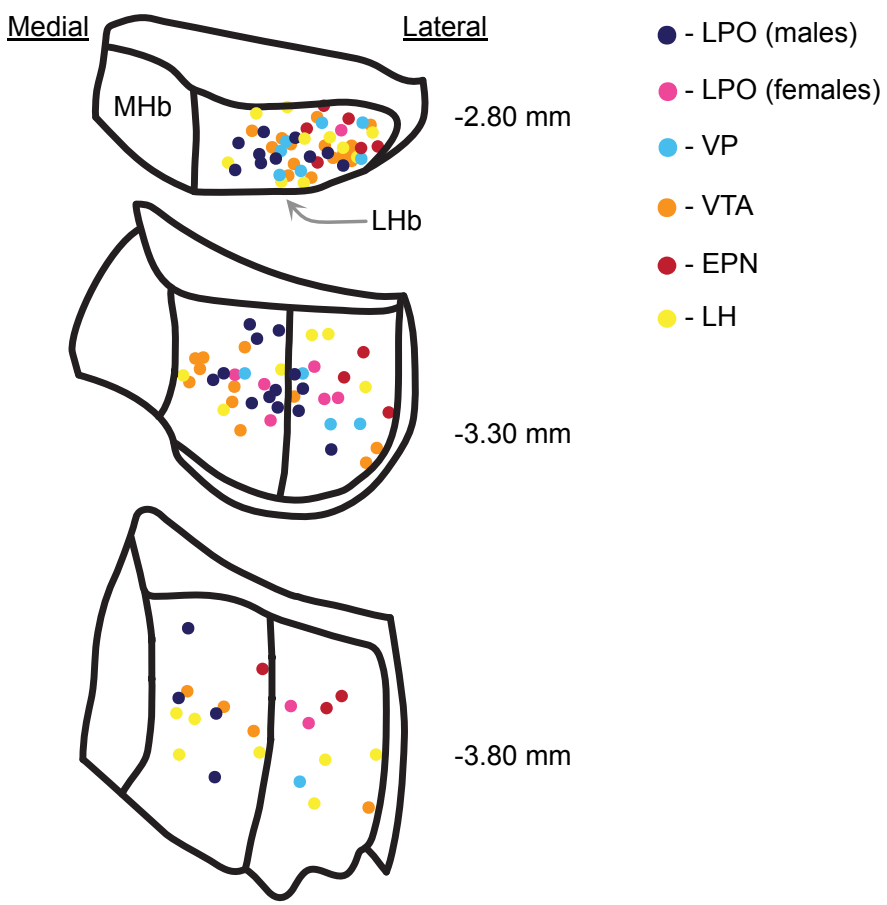

Extended Data Figure 2. Neurons receiving functional synaptic inputs from the five brain regions showing connectivity were distributed throughout the LHb. Locations of neurons found connected using whole-cell recordings from optogenetic stimulation of terminals arising from each input tested. Locations are based on biocytin immunohistochemistry and low magnification images taken on the recording microscope where the recorded cell is centered within the field of view. Color indicates source of innervation. 
Extended Figure 3

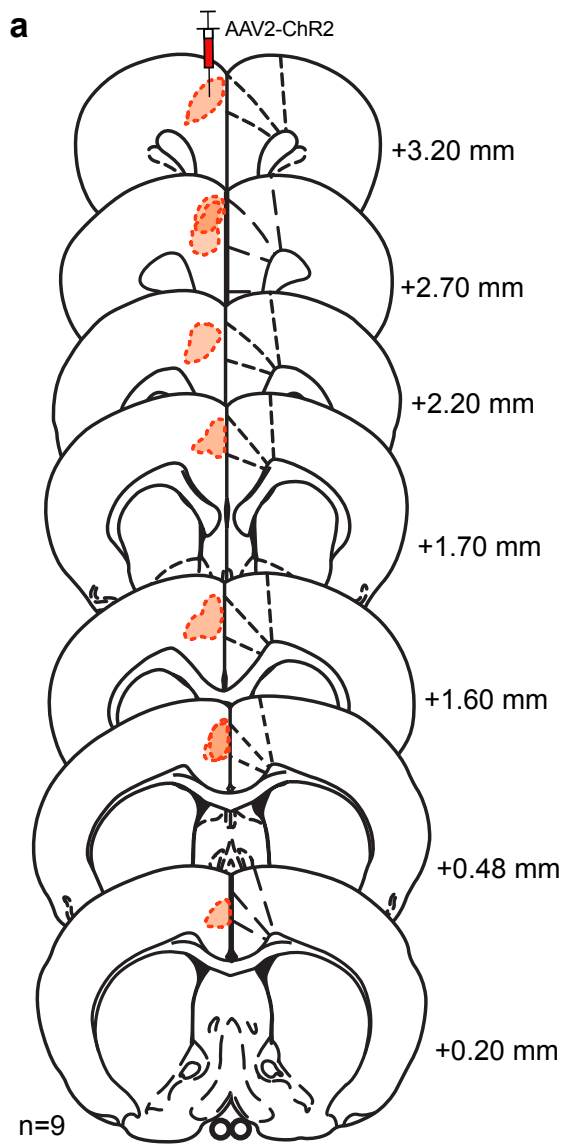

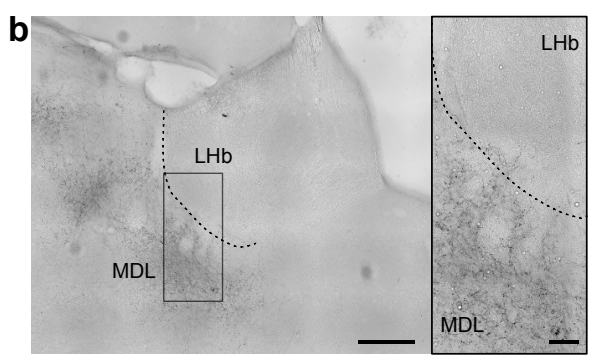

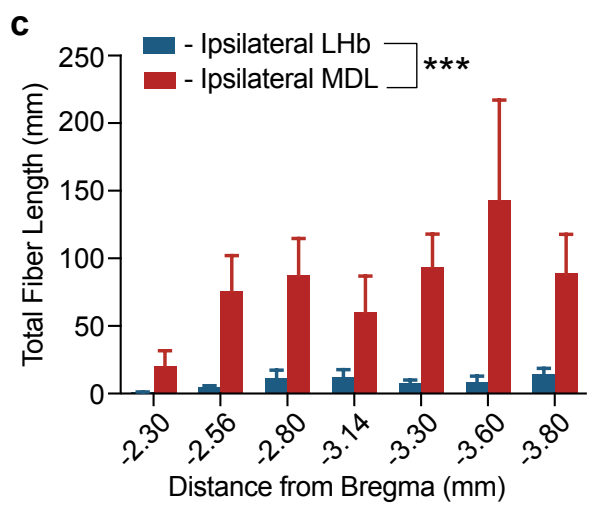

Extended Data Figure 3. ACC minimally innervates the $L H b$. a, Diagram of the extent of unilateral AAV2-hSynhChR2(H134R)-mCherry injection sites (n $=9$ male rats) throughout anteroposterior range of the ACC for anterograde tracing study. b, Example ipsilateral DAB-positive fibers (black) visualized under brightfield illumination. Fibers heavily innervate the MDL, which abuts the lateral edge of the $\mathrm{LHb}$, while sparse to no fibers innervate the $\mathrm{LHb}$. Contralateral innervation of $\mathrm{LHb}$ and MDL was negligible compared to the ipsilateral side, and therefore were omitted from our analysis. (Left) Scale bar $=250$ $\mu \mathrm{m}$. (Right) Scale bar $=50 \mu \mathrm{m}$. c, Average stereologically-quantified DAB-positive fiber lengths throughout anteroposterior range of the $\mathrm{LHb}$, compared to the MDL innervation in the same coronal slice. Mann-Whitney test, two-tailed: $\mathrm{U}=0, \mathrm{p}=$ 0.0006 . 


\section{Extended Data Figure 4}
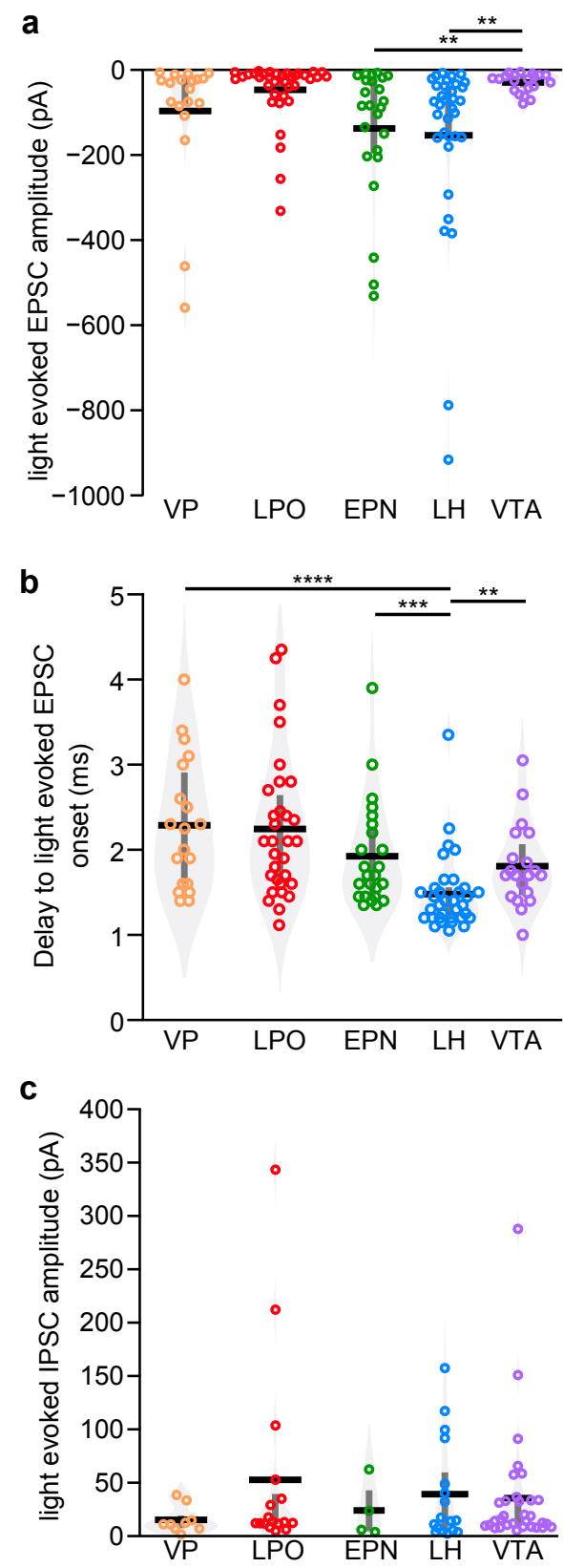

Extended Data Figure 4. Sources of glutamatergic inputs to the rat LHb vary in strength and delay in synaptic transmission. a, We observed some variations between inputs in the mean light evoked EPSCs $\left(\mathrm{V}_{\mathrm{m}}=-60 \mathrm{mV}\right)$. In particular, excitatory inputs from the VTA were consistently small: Kruskal-Wallis $\chi 2=$ $25.5, \mathrm{df}=4, \mathrm{p}=0.00004$, followed by Dunn Test for pairwise comparisons. $\mathbf{b}$, Differences were also detected in the delay to the onset of light evoked EPSCs to $\mathrm{LHb}$ neurons from these different sources, with inputs from LH showing the fastest response. Although these data deviate from a normal distribution (Shapiro's test, $p=0.0000001$ ), KDEs (violins) are consistent with continuous distributions, suggesting reliable polysynaptic events were rarely detected. c, We observed some variations between inputs in the mean light evoked IPSCs $\left(\mathrm{V}_{\mathrm{m}}=\right.$ $40 \mathrm{mV}$ ). While there were no statistically significant differences in amplitudes detected, the mean inhibitory input from the VP was particularly small: KruskalWallis $\chi^{2}=2.3$, df $=4, \mathrm{p}=0.7$. ${ }^{* *} \mathrm{p}<0.01,{ }^{* * *} \mathrm{p}<0.005, * * * * \mathrm{p}<0.0005$ 

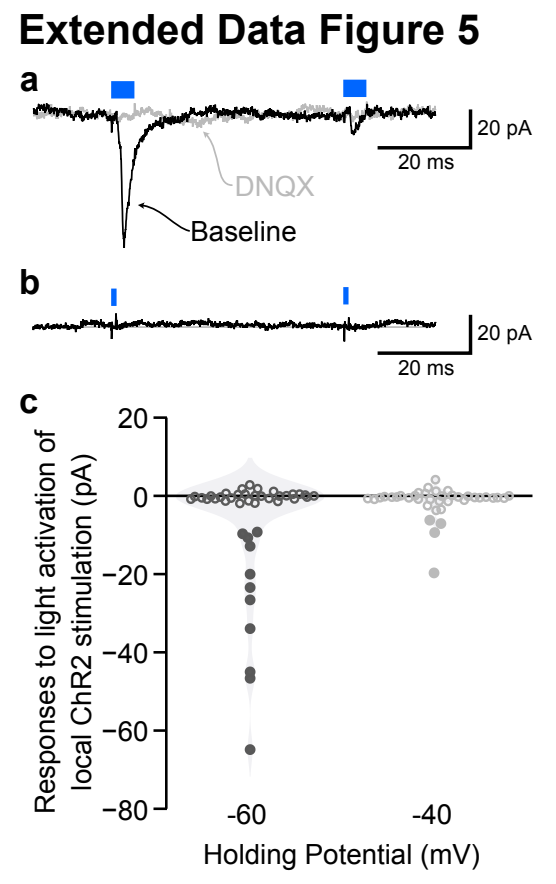

Extended Data Figure 5. Optogenetic experiments detect local glutamate, but not GABA, functional connections in rat LHb. AAV2-hSyn-hChR2(H134R)-mCherry was stereotaxically injected into the LHb at least 4 weeks prior to ex vivo whole cell recordings in the $\mathrm{LHb}$ to detect local synaptic connections. Neurons were recorded blind to ChR2 expression, therefore in some cases the patched neuron expressed ChR2. Therefore, in addition to connectivity criteria used for other afferent inputs, in these experiments only light evoked inward currents that were blocked by $10 \mu \mathrm{M}$ DNQX were considered glutamatergic connections, and direct ChR2 induced inward currents were subtracted out for the quantification illustrated here. Each cell was probed for both glutamate and GABA inputs in voltage clamp by holding neurons at $\mathrm{V}_{\mathrm{m}}=-60 \mathrm{mV}$ and $-40 \mathrm{mV}$, light pulse durations 1,5 , and $10 \mathrm{~ms}$ durations. a, Example recording at $\mathrm{V}_{\mathrm{m}}=-60 \mathrm{mV}$ showing a light evoked response that was blocked by DNQX. b, Example recording at $\mathrm{V}_{\mathrm{m}}=-40 \mathrm{mV}$ with minimal outward current response within $7 \mathrm{~ms}$ of light pulse. c, Summary of all LHb recordings tested in this experiment. Filled circles represent cells where responses could be classified as local $\mathrm{ChR} 2$ induced synaptic transmission. When no clear response was detected, the measure indicated is the difference between the mean $I_{\text {holding }}$ of the baseline $100 \mathrm{~ms}$ period just prior to the light pulse and the mean $I_{\text {holding }} 2 \mathrm{~ms}$ period

starting $2 \mathrm{~ms}$ after initiation of the light pulse, consistent with monosynaptic delay timing. None of the recordings made at $\mathrm{V}_{\mathrm{m}}=-40 \mathrm{mV}$ met the criteria for a response to light stimulation with an outward current. 


\section{Extended Data Figure 6}

a $\begin{array}{lll}\text { Baseline } & 500 \mathrm{nM} \text { TTX } & 500 \mathrm{nM} \\ & 100 \mu \mathrm{M} 4 \mathrm{AP} & \text { DAMGO }\end{array}$

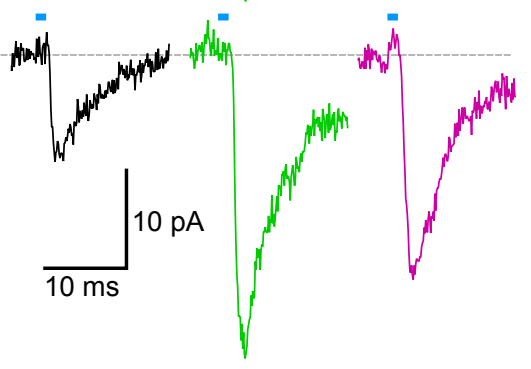

b

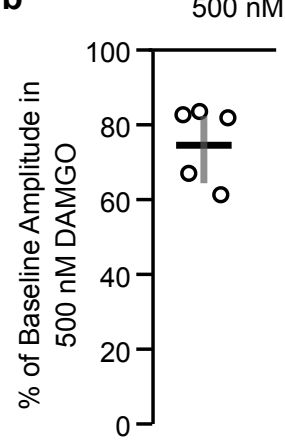

500 nM TTX \& $100 \mu \mathrm{M} 4$ AP

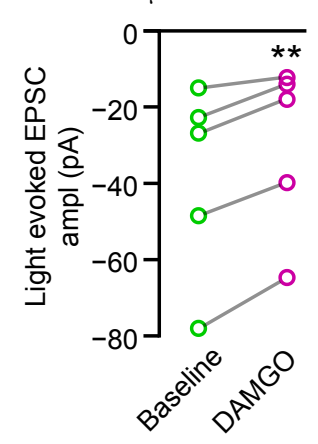

Extended Data Figure 6. Isolated

monosynaptic glutamatergic inputs from LPO to LHb neurons are inhibited by MOR activation. a, Example light evoked EPSC responses in an $\mathrm{LHb}$ neuron from a rat with ChR2 expression in LPO neurons. This response persisted in monosynaptic isolation by $500 \mathrm{nM}$ TTX and $100 \mu \mathrm{M} 4 \mathrm{AP}$ (green), and this isolated response was inhibited by 500 nM DAMGO (magenta). b, Summary of

DAMGO effects on isolated monosynaptic EPSC inputs to LHb neurons expressed as \% of baseline monosynaptic response (left) and as raw EPSC magnitudes (right). Paired t-test, $\mathrm{df}=4, \mathrm{t}=-5.1, \mathrm{p}=0.007$. ${ }^{* *} \mathrm{p}<0.01$ 


\section{Extended Data Figure 7}

a

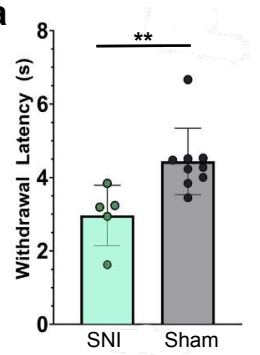

b

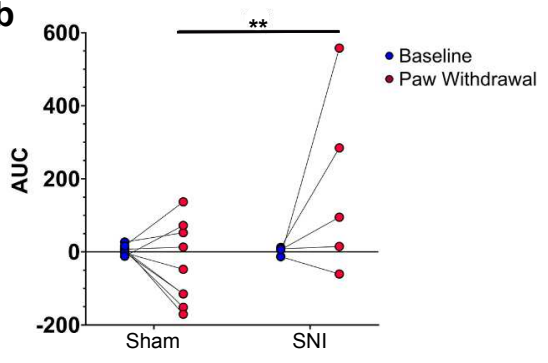

Extended Data Figure 7. Mice with SNI show hypersensitivity to heat and increased activity in LHb-projecting LPO neurons during paw withdrawal from thermal stimulation. a, Mice with SNI show a reduced latency to withdraw their paw following thermal stimulation in the Hargreaves task. Unpaired t-test, $\mathrm{t}(12)=3.007, \mathrm{p}=0.011 . \mathbf{b}$, VGluT2-expressing LPO neurons that project to the $\mathrm{LHb}$ expressed GCaMP6m and showed a greater calcium response during paw withdrawal to Hargreaves

thermal stimulation (area under the curve, deviation from baseline fluorescence) in SNI animals $(\mathrm{n}=5)$ compared to sham controls $(n=9)$ : Two-way ANOVA, $F(1,12)=5.4, p=0.038$; Holm-Sidak post-hoc test, $p=0.0074 .{ }^{* *} p<0.01$ 


\section{Extended Data Figure 8}

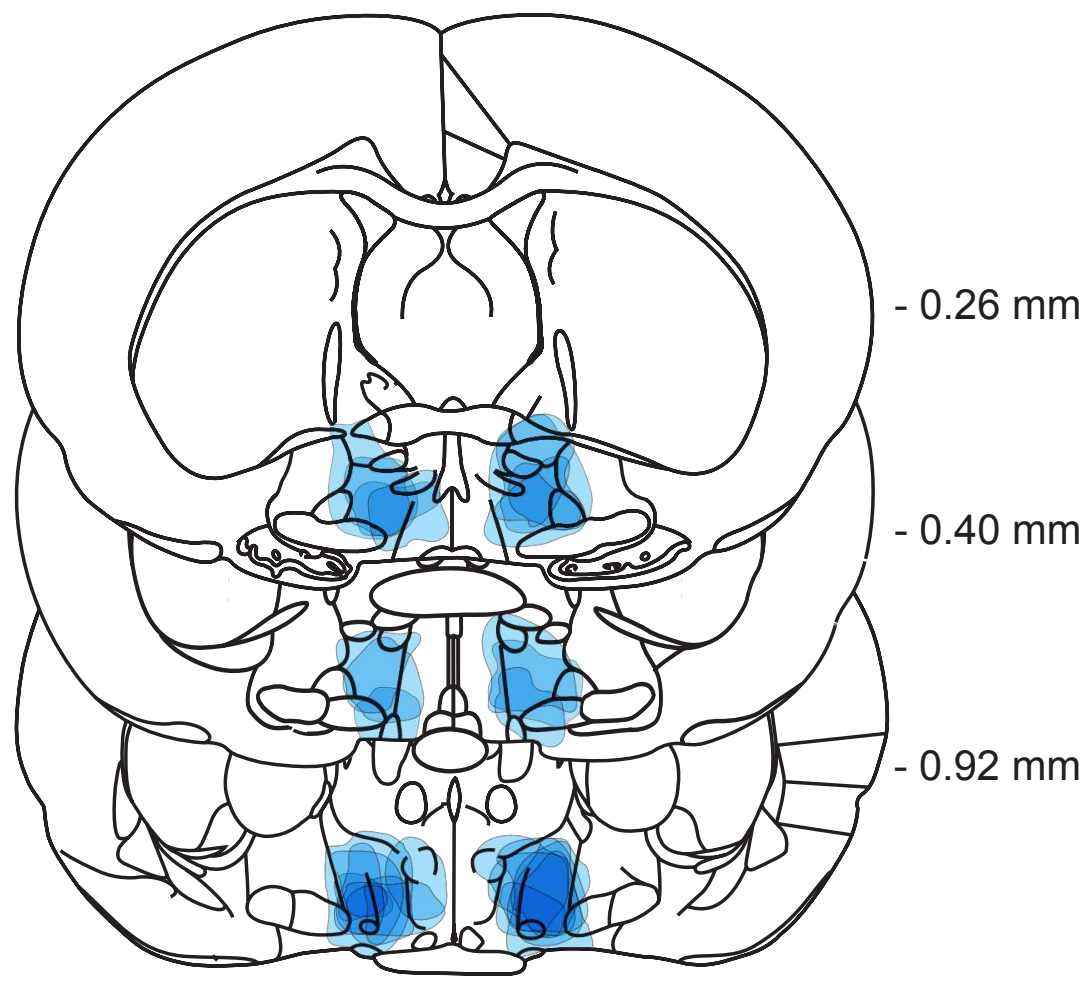

Extended Data Figure 8. Locations and spread of bilateral ChR2 injections to the LPO in rats used for in vivo optogenetic experiments. 


\section{Supplementary Table 1: Assumption testing on behavioral data}

\begin{tabular}{|c|c|c|c|c|c|c|}
\hline Experiment & Figure & $\begin{array}{l}\text { \# animals; } \\
\text { \# Outliers; } \\
\text { \# extreme } \\
\text { outliers }\end{array}$ & $\begin{array}{l}\text { Shapiro-Wilk } \\
\text { Test of } \\
\text { Normality } \\
\text { (across all } \\
\text { groups) } \\
\text { Statistic; p val }\end{array}$ & $\begin{array}{l}\text { Test for } \\
\text { Homogeneity of } \\
\text { Variances } \\
\text { Statistic; p val } \\
\text { (Test used) }\end{array}$ & Parametric test & $\begin{array}{l}\text { Non-parametric test: } \\
\text { Repeated measures } \\
\text { Wilcoxon signed rank } \\
\text { exact test } \\
\mathrm{V} ; \text { p val }\end{array}$ \\
\hline $\begin{array}{l}\text { von Frey: Male; Sham; } \\
10 \mu \mathrm{M} \text { DAMGO in } \\
\text { LHb }\end{array}$ & $1 \mathrm{~b}$ & $9 ; 0 ; 0$ & $0.773 ; 0.000638$ & $\begin{array}{l}0.0760 ; 0.786 \\
\text { (Levene's Test) }\end{array}$ & $\mathrm{n} / \mathrm{a}$ & $1.5 ; 1$ \\
\hline $\begin{array}{l}\text { von Frey: Male; SNI; } \\
10 \mu \mathrm{M} \text { DAMGO in } \\
\mathrm{LHb}\end{array}$ & $1 \mathrm{~b}$ & $8 ; 0 ; 0$ & $0.866 ; 0.0240$ & $\begin{array}{l}1.49 ; 0.242 \\
\text { (Levene's Test) }\end{array}$ & $\mathrm{n} / \mathrm{a}$ & $2 ; 0.05024$ \\
\hline $\begin{array}{l}\text { von Frey: Female; } \\
\text { SNI; } 10 \mu \mathrm{M} \text { DAMGO } \\
\text { in LHb }\end{array}$ & $1 \mathrm{~b}$ & $9 ; 2 ; 1$ & $0.805 ; 0.00181$ & $\begin{array}{l}1.82 ; 0.196 \\
\text { (Levene's Test) }\end{array}$ & $\mathrm{n} / \mathrm{a}$ & $7 ; 0.07422$ \\
\hline $\begin{array}{l}\text { von Frey: Male; Sham; } \\
10 \mu \text { M DAMGO in } \\
\text { i.c.v. }\end{array}$ & $1 \mathrm{~b}$ & $9 ; 0 ; 0$ & $0.768 ; 0.00055$ & $\begin{array}{l}0.0897 ; 0.768 \\
\text { (Levene's Test) }\end{array}$ & $\mathrm{n} / \mathrm{a}$ & $7 ; 0.5294$ \\
\hline $\begin{array}{l}\text { von Frey: Male; SNI; } \\
10 \mu \mathrm{M} \text { DAMGO in } \\
\text { i.c.v. }\end{array}$ & $1 \mathrm{~b}$ & $12 ; 2 ; 2$ & $\begin{array}{l}0.756 ; \\
0.0000618\end{array}$ & $\begin{array}{l}0.168 ; 0.686 \\
\text { (Levene's Test) }\end{array}$ & $\mathrm{n} / \mathrm{a}$ & $31 ; 0.08006$ \\
\hline $\begin{array}{l}\text { Place Conditioning: } \\
\text { Male Sham/SNI; } 10 \\
\mu \text { M DAMGO in LHb }\end{array}$ & $1 \mathrm{c}$ & $15 ; 4 ; 0$ & $\begin{array}{l}\text { Sham x } \\
\text { Baseline: } 0.95 \text {; } \\
0.726 \\
\text { SNI x Baseline: } \\
0.94 ; 0.686 \\
\text { Sham x Test: } \\
0.936 ; 0.542 \\
\text { SNI x Test: } \\
0.840 ; 0.129\end{array}$ & $\begin{array}{l}4.68 ; 0.0305 \\
\text { (Box M-test) }\end{array}$ & $\begin{array}{l}\text { Two-way mixed design ANOVA } \\
\text { two-way interaction } \mathrm{F}(1,13)=15.932 ; \mathrm{p} \\
=0.002 \\
\text { effect of group on baseline: } \mathrm{F}=0.293 \\
\text { adjusted } \mathrm{p}=1 \\
\text { effect of group on test day: } \mathrm{F}=11.3 ; \\
\text { adjusted } \mathrm{p}=0.01 \\
\text { Paired t-tests, adjusted } \\
\text { Sham: adjusted } \mathrm{p}=1 \\
\text { SNI: adjusted } \mathrm{p}=0.012\end{array}$ & $\mathrm{n} / \mathrm{a}$ \\
\hline $\begin{array}{l}\text { Place Conditioning: } \\
\text { Sham vs. Male SNI; } \\
10 \mu \mathrm{M} \text { DAMGO in } \\
\text { i.c.v. }\end{array}$ & $1 \mathrm{c}$ & $21 ; 2 ; 0$ & $\begin{array}{l}\text { Sham x } \\
\text { Baseline: } 0.901 \text {; } \\
0.257 \\
\text { SNI x Baseline: } \\
0.967 ; 0.872 \\
\text { Sham x Test: } \\
0.971 ; 0.905 \\
\text { SNI x Test: } \\
0.985 ; 0.997 \\
\end{array}$ & $\begin{array}{l}23.9 ; \\
0.000000997 \\
\text { (Box M-test) }\end{array}$ & $\begin{array}{l}\text { Two-way mixed design ANOVA } \\
\text { two-way interaction } \mathrm{F}(1,19)=2.239 ; \mathrm{p}= \\
0.151 \\
\text { Paired t-tests for non-significant two- } \\
\text { way interaction: } \\
\text { Sham: adjusted } \mathrm{p}=0.286 \\
\text { SNI: adjusted } \mathrm{p}=0.433\end{array}$ & $\mathrm{n} / \mathrm{a}$ \\
\hline $\begin{array}{l}\text { von Frey: Female; } \\
\text { Sham; } 100 \mu \mathrm{M} \\
\text { DAMGO in LHb }\end{array}$ & $1 \mathrm{~d}$ & $6 ; 0 ; 0$ & $0.838 ; 0.026$ & $\begin{array}{l}0.493 ; 0.499 \\
\text { (Levene's Test) }\end{array}$ & $\mathrm{n} / \mathrm{a}$ & $5 ; 1$ \\
\hline $\begin{array}{l}\text { von Frey: Female; } \\
\text { SNI; } 100 \mu \mathrm{M} \\
\text { DAMGO in LHb }\end{array}$ & $1 \mathrm{~d}$ & $9 ; 1 ; 1$ & $0.839 ; 0.00928$ & $\begin{array}{l}1.13 ; 0.306 \\
\text { (Levene's Test) }\end{array}$ & $\mathrm{n} / \mathrm{a}$ & $6 ; 0.4017$ \\
\hline $\begin{array}{l}\text { Place Conditioning: } \\
\text { Female Sham/SNI; } \\
100 \mu \mathrm{M} \text { DAMGO in } \\
\mathrm{LHb}\end{array}$ & $1 \mathrm{e}$ & $15 ; 3 ; 0$ & $\begin{array}{l}\text { Sham x } \\
\text { Baseline: } 0.904 \\
0.66 \\
\text { SNI x Baseline: } \\
0.919 ; 0.384 \\
\text { Sham x Test: } \\
0.87 ; 0.226 \\
\text { SNI x Test: } \\
0.900 ; 0.250\end{array}$ & $\begin{array}{l}8.59 ; 0.00339 \\
\text { (Box M-test) }\end{array}$ & $\begin{array}{l}\text { Two-way mixed design ANOVA } \\
\text { two-way interaction } \mathrm{F}(1,13)=6.234 ; \mathrm{p}= \\
0.027 \\
\text { effect of group on baseline: } \mathrm{F}=0.006 ; \\
\text { adjusted } \mathrm{p}=1 \\
\text { effect of group on test day: } \mathrm{F}=5.56 ; \\
\text { adjusted } \mathrm{p}=0.07 \\
\text { Paired t-tests, adjusted } \\
\text { Sham: adjusted } \mathrm{p}=0.476 \\
\text { SNI: adjusted } \mathrm{p}=0.0349\end{array}$ & $\mathrm{n} / \mathrm{a}$ \\
\hline $\begin{array}{l}\text { Fiber photometry; } \\
\text { Sham vs. SNI mice }\end{array}$ & $5 b$ & $\begin{array}{l}14 ; \text { not } \\
\text { determined }\end{array}$ & not determined & not determined & $\begin{array}{l}\text { Two-way mixed design ANOVA } \\
\mathrm{F}(1,12)=5.439 ; \\
\mathrm{p}=0.038 ; \\
\text { Holm-Sidak post-hoc test, } \mathrm{p}=0.0074\end{array}$ & $\mathrm{n} / \mathrm{a}$ \\
\hline $\begin{array}{l}\text { Place Conditioning; } \\
\text { Male; mCherry vs. } \\
\text { ChR2; } 10 \mu \mathrm{M} \\
\text { DAMGO in LHb }\end{array}$ & $5 \mathrm{e}$ & $16 ; 0 ; 0$ & $\begin{array}{l}\text { mCherry } \mathrm{x} \\
\text { Baseline: } 0.941 ; \\
0.618 \\
\text { ChR2 x } \\
\text { Baseline: } 0.913 ; \\
0.374\end{array}$ & $\begin{array}{l}\text { 18.3; } 0.0000186 \\
\text { (Box M-test) }\end{array}$ & $\begin{array}{l}\text { Two-way mixed design ANOVA } \\
\text { two-way interaction, } \mathrm{F}(1,14)=9.982 ; \mathrm{p} \\
=0.007 \\
\text { effect of group on baseline: } \mathrm{F}=0.01 \\
\text { adjusted } \mathrm{p}=1\end{array}$ & $\mathrm{n} / \mathrm{a}$ \\
\hline
\end{tabular}




\begin{tabular}{|c|c|c|c|c|c|c|}
\hline & & & $\begin{array}{l}\text { mCherry } \mathrm{x} \text { Test: } \\
0.914 ; 0.385 \\
\text { ChR2 } \mathrm{x} \text { Test: } \\
0.847 ; 0.0891\end{array}$ & & $\begin{array}{l}\text { effect of group on test day: } \mathrm{F}=12.5 \\
\text { adjusted } \mathrm{p}=0.006 \\
\text { Paired t-tests, adjusted } \\
\text { mCherry: adjusted } \mathrm{p}=0.427 \\
\text { ChR2: adjusted } \mathrm{p}=0.0027\end{array}$ & \\
\hline $\begin{array}{l}\text { Hargreaves: Male; } \\
\text { Sham; } 10 \mu \mathrm{M} \\
\text { DAMGO in LHb }\end{array}$ & ED 1a & $9 ; 2 ; 0$ & $0.966 ; 0.726$ & $\begin{array}{l}\text { Bartlett's K- } \\
\text { squared }= \\
0.035658, \mathrm{df}=1, \\
\text { p-value }=0.8502 \\
\text { (Bartlett Test) } \\
\end{array}$ & $\begin{array}{l}\text { Paired t-test } \\
\mathrm{Df}=8 ; \mathrm{t}=1.06 ; \mathrm{p}=0.32\end{array}$ & $\mathrm{n} / \mathrm{a}$ \\
\hline $\begin{array}{l}\text { Hargreaves; Male; } \\
\text { SNI; } 10 \mu \mathrm{M} \text { DAMGO } \\
\text { in LHb }\end{array}$ & ED 1a & $8 ; 0 ; 0$ & $0.941 ; 0.365$ & $\begin{array}{l}\text { Bartlett's K- } \\
\text { squared }=3.704 \\
\mathrm{df}=1, \text { p-value }= \\
0.096 \text { (Bartlett } \\
\text { Test) }\end{array}$ & $\begin{array}{l}\text { Paired t-test } \\
\mathrm{Df}=7 ; \mathrm{t}=-1.92 ; \mathrm{p}=0.096\end{array}$ & $\mathrm{n} / \mathrm{a}$ \\
\hline $\begin{array}{l}\text { Hargreaves; Female; } \\
\text { SNI; } 10 \mu \mathrm{M} \text { DAMGO } \\
\text { in LHb }\end{array}$ & ED 1a & $9 ; 1 ; 1$ & $0.944 ; 0.333$ & $\begin{array}{l}\text { Bartlett's K- } \\
\text { squared }=1.35 \mathrm{df} \\
=1, \mathrm{p} \text {-value }= \\
0.245 \text { (Bartlett } \\
\text { Test) }\end{array}$ & $\begin{array}{l}\text { Paired t-test } \\
\mathrm{Df}=8 ; \mathrm{t}=-1.4 ; \mathrm{p}=0.199\end{array}$ & $\mathrm{n} / \mathrm{a}$ \\
\hline $\begin{array}{l}\text { Hargreaves: Male; } \\
\text { Sham; } 10 \mu \mathrm{M} \\
\text { DAMGO in i.c.v. }\end{array}$ & ED 1a & $9 ; 3 ; 0$ & $0.927 ; 0.174$ & $\begin{array}{l}0.840 ; 0.373 \\
\text { (Levene's Test) }\end{array}$ & $\begin{array}{l}\text { Paired t-test } \\
\mathrm{Df}=8 ; \mathrm{t}=-0.08 ; \mathrm{p}=0.938\end{array}$ & $\mathrm{n} / \mathrm{a}$ \\
\hline $\begin{array}{l}\text { Hargreaves; Male; } \\
\text { SNI; } 10 \mu \mathrm{M} \text { DAMGO } \\
\text { in i.c.v. }\end{array}$ & ED 1a & $8 ; 0 ; 0$ & $0.941 ; 0.365$ & $\begin{array}{l}0.013 ; 0.911 \\
\text { (Levene's Test) }\end{array}$ & $\begin{array}{l}\text { Paired t-test } \\
\mathrm{Df}=11 ; \mathrm{t}=3.2 ; \mathrm{p}=0.00846\end{array}$ & $\mathrm{n} / \mathrm{a}$ \\
\hline $\begin{array}{l}\text { von Frey; Male; Sham; } \\
10 \mu \mathrm{M} \text { DAMGO in } \\
\text { LHb }\end{array}$ & ED 1c & $9 ; 0 ; 0$ & $0.773 ; 0.000638$ & $\begin{array}{l}0.0760 ; 0.786 \\
\text { (Levene's Test) }\end{array}$ & $\mathrm{n} / \mathrm{a}$ & $1.5 ; 1$ \\
\hline $\begin{array}{l}\text { von Frey; Male; CFA; } \\
10 \mu \mathrm{M} \text { DAMGO in } \\
\mathrm{LHb}\end{array}$ & ED 1c & $9 ; 0 ; 0$ & $0.903 ; 0.0639$ & $\begin{array}{l}3.800 .0691 \\
\text { (Levene's Test) }\end{array}$ & $\begin{array}{l}\text { Paired t-test } \\
\mathrm{Df}=8 ; \mathrm{t}=-2.89 ; \mathrm{p}=0.0202\end{array}$ & $\mathrm{n} / \mathrm{a}$ \\
\hline $\begin{array}{l}\text { Hargreaves; Male; } \\
\text { Sham; } 10 \mu \mathrm{M} \\
\text { DAMGO in LHb }\end{array}$ & ED 1d & $9 ; 2 ; 0$ & $0.966 ; 0.726$ & $\begin{array}{l}\text { Bartlett's K- } \\
\text { squared }= \\
0.035658, \mathrm{df}=1, \\
\text { p-value }=0.8502 \\
\text { (Bartlett Test) } \\
\end{array}$ & $\begin{array}{l}\text { Paired t-test } \\
\mathrm{Df}=8 ; \mathrm{t}=1.06 ; \mathrm{p}=0.32\end{array}$ & $\mathrm{n} / \mathrm{a}$ \\
\hline $\begin{array}{l}\text { Hargreaves; Male; } \\
\text { CFA; } 10 \mu \mathrm{M} \text { DAMGO } \\
\text { in LHb }\end{array}$ & ED 1d & $9 ; 2 ; 2$ & $0.858 ; 0.0113$ & $\begin{array}{l}0.04610 .833 \\
\text { (Levene's Test) }\end{array}$ & $\mathrm{n} / \mathrm{a}$ & $12 ; 0.25$ \\
\hline $\begin{array}{l}\text { Place Conditioning; } \\
\text { Male; Sham vs. CFA; } \\
10 \mu \text { M DAMGO in } \\
\text { LHb }\end{array}$ & ED 1e & $16 ; 3 ; 0$ & $\begin{array}{l}\text { Sham x } \\
\text { Baseline: } 0.901 \text {; } \\
0.257 \\
\text { CFA x Baseline: } \\
0.94 ; 0.635 \\
\text { Sham x Test: } \\
0.971 ; 0.905 \\
\text { CFA x Test: } \\
0.828 ; 0.0774 \\
\end{array}$ & $\begin{array}{l}\text { 13.4; } 0.000253 \\
\text { (Box M-test) }\end{array}$ & $\begin{array}{l}\text { Two-way mixed design ANOVA } \\
\text { two-way interaction } \mathrm{F}(1,14)=0.474 ; \mathrm{p}= \\
0.502 \\
\text { Paired t-tests for non-significant two- } \\
\text { way interaction: } \\
\text { Sham: adjusted } \mathrm{p}=0.286 \\
\text { CFA: adjusted } \mathrm{p}=0.761\end{array}$ & $\mathrm{n} / \mathrm{a}$ \\
\hline $\begin{array}{l}\text { ACC fiber innervation; } \\
\text { Ipsi LHb vs. Ipsi MDL }\end{array}$ & ED 3c & $9 ; 0 ; 0$ & not determined & not determined & $\mathrm{n} / \mathrm{a}$ & $\begin{array}{l}\text { Mann-Whitney test, two- } \\
\text { tailed, } U=0 ; p=0.0006\end{array}$ \\
\hline $\begin{array}{l}\text { Hargreaves; Sham vs. } \\
\text { SNI mice }\end{array}$ & ED 7a & $14 ; 3 ; 2$ & $0.896 ; 0.0994$ & $\begin{array}{l}0.0012 ; 0.973 \\
\text { (Levene's Test) }\end{array}$ & $\begin{array}{l}\text { Unpaired t-test } \\
\mathrm{Df}=12, \mathrm{t}=3.007, \mathrm{p}=0.0109\end{array}$ & $\mathrm{n} / \mathrm{a}$ \\
\hline $\begin{array}{l}\text { AUC summary: } \\
\text { Hargreaves/Fiber } \\
\text { photometry; Sham vs. } \\
\text { SNI mice }\end{array}$ & ED 7b & $14 ; 0 ; 0$ & $\begin{array}{l}\text { Sham x } \\
\text { Baseline: } 0.979 \text {; } \\
0.257 \\
\text { Sham x paw } \\
\text { withdrawal: } \\
0.932 ; 0.496 \\
\text { SNI x Baseline: } \\
0.813 ; 0.103 \\
\text { SNI x paw } \\
\text { withdrawal: } \\
0.921 ; 0.538 \\
\end{array}$ & $\begin{array}{l}\text { 53.4; } 2.71 \mathrm{e}-13 \\
\text { (Box M-test) }\end{array}$ & $\begin{array}{l}\text { Two-way mixed design ANOVA } \\
\mathrm{F}(1,12)=5.439 ; \mathrm{p}=0.038 ; \text { Holm-Sidak } \\
\text { post-hoc test, } \mathrm{p}=0.0074\end{array}$ & $\mathrm{n} / \mathrm{a}$ \\
\hline
\end{tabular}




\section{Figures}

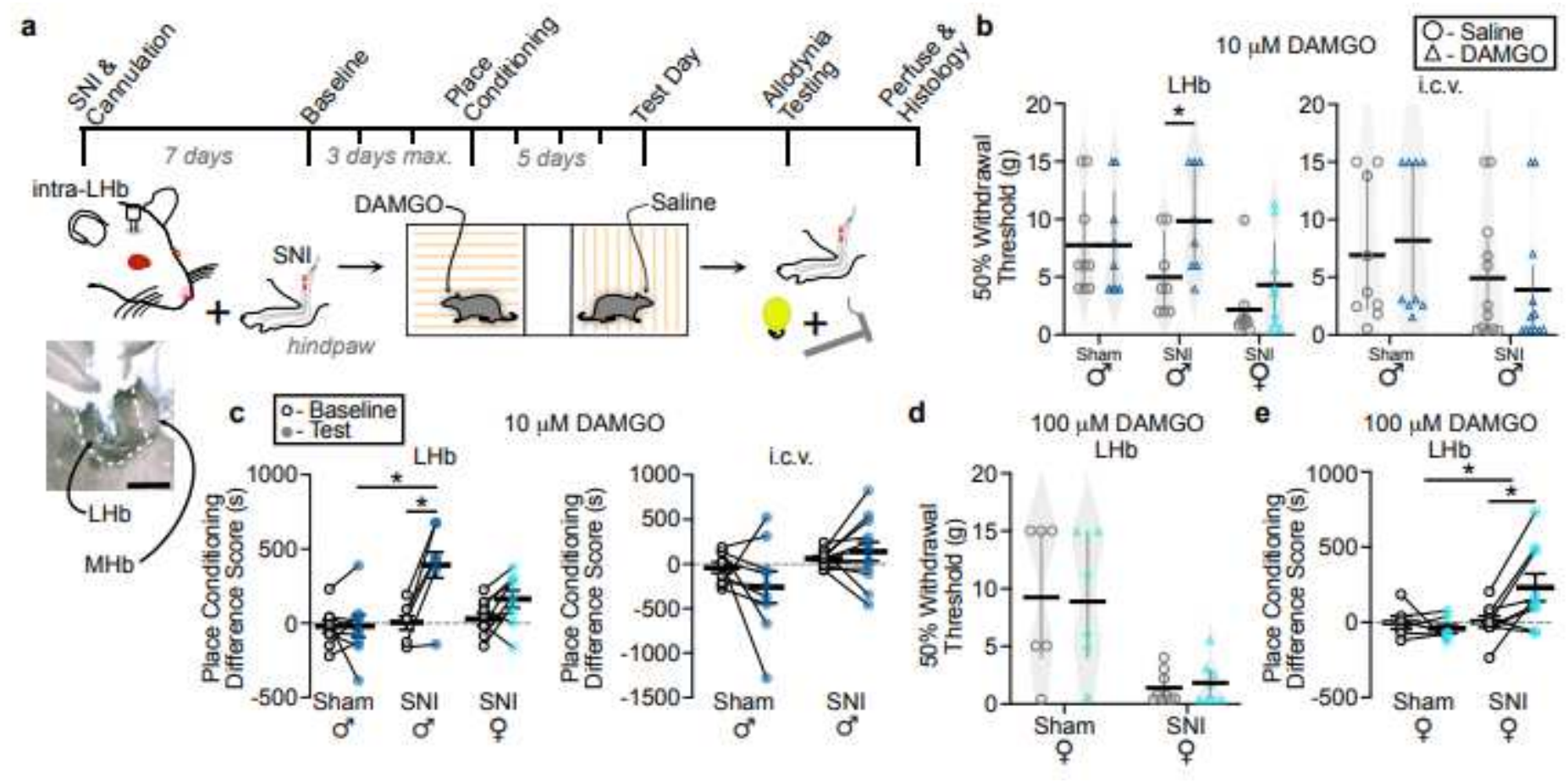

\section{Figure 1}

MOR activation in the LHb relieves allodynia and generates negative reinforcement in rats with neuropathic pain. a, Timeline of behavioral experiments. Lower inset, example injection site centered in LHb. Scale bar $=500 \mu \mathrm{m}$. b, (Left) Violin plots of $50 \%$ mechanical withdrawal thresholds following saline or $10 \mu$ M DAMGO microinjections into the LHb in sham males $(n=9)$, SNI males $(n=8)$, and SNI females $(n=9)$. Sham males: Wilcoxon signed rank test, $V=1.5, p=1$. SNI males: Wilcoxon signed rank test, $V=2$, $p=0.050$. Females trended towards an increase in threshold following DAMGO infusion: Wilcoxon signed rank test, $V=7, p=0.074$. (Right) Mechanical withdrawal thresholds in male rats with i.c.v. cannulae, with SNI $(n=12)$ or sham $(n=9)$ injuries. Sham: Wilcoxon signed rank test, $V=7, p=0.53$. SNI: Wilcoxon signed rank test, $V=31, p=0.080$. $c$, Difference scores before and after conditioning with intracranial microinjections. (Left) Males with SNI developed a preference for the chamber paired with intra-LHb DAMGO. Twoway mixed ANOVA, significant interaction between SNI/sham and baseline/test $F(1,13)=$ $15.93, p=0.002$; post hoc effect group on test day adjusted $p=0.01$; paired t-tests, sham adjusted $p=1$; $\mathrm{SNI}$ adjusted $p=0.016$. Female $\mathrm{SNI}$ animals trended towards a preference for the DAMGO-paired chamber: Paired t-test $p=0.054$. (Right) i.c.v. DAMGO did not produce a preference in sham or SNI male rats: Two-way mixed ANOVA, $F(1,19)=2.239, p=0.15$. $d$, Mechanical withdrawal thresholds in sham $(n=$ 6) and SNI $(n=9)$ female rats following $100 \mu$ M DAMGO infusions into the LHb. Sham: Wilcoxon signed rank test, $V=5, p=1$. SNl: Wilcoxon signed rank test, $V=6, p=0.40$. e, Place conditioning of sham and SNI females to intra-LHb $100 \mu \mathrm{M}$ DAMGO vs saline. Females with SNI developed a significant CPP:

Twoway mixed ANOVA, significant interaction between SNI/sham and baseline/test $F(1,13)=6.234, p=$ 0.027 ; post hoc effect group on test day adjusted $p=0.07$; paired t-tests, sham adjusted $p=0.48$; SNI 
adjusted $p=0.035$. Means represented by horizontal lines; SEM and quartiles indicated by vertical lines with and without caps, respectively. ${ }^{*} p \leq 0.05,{ }^{* *} p<0.01$
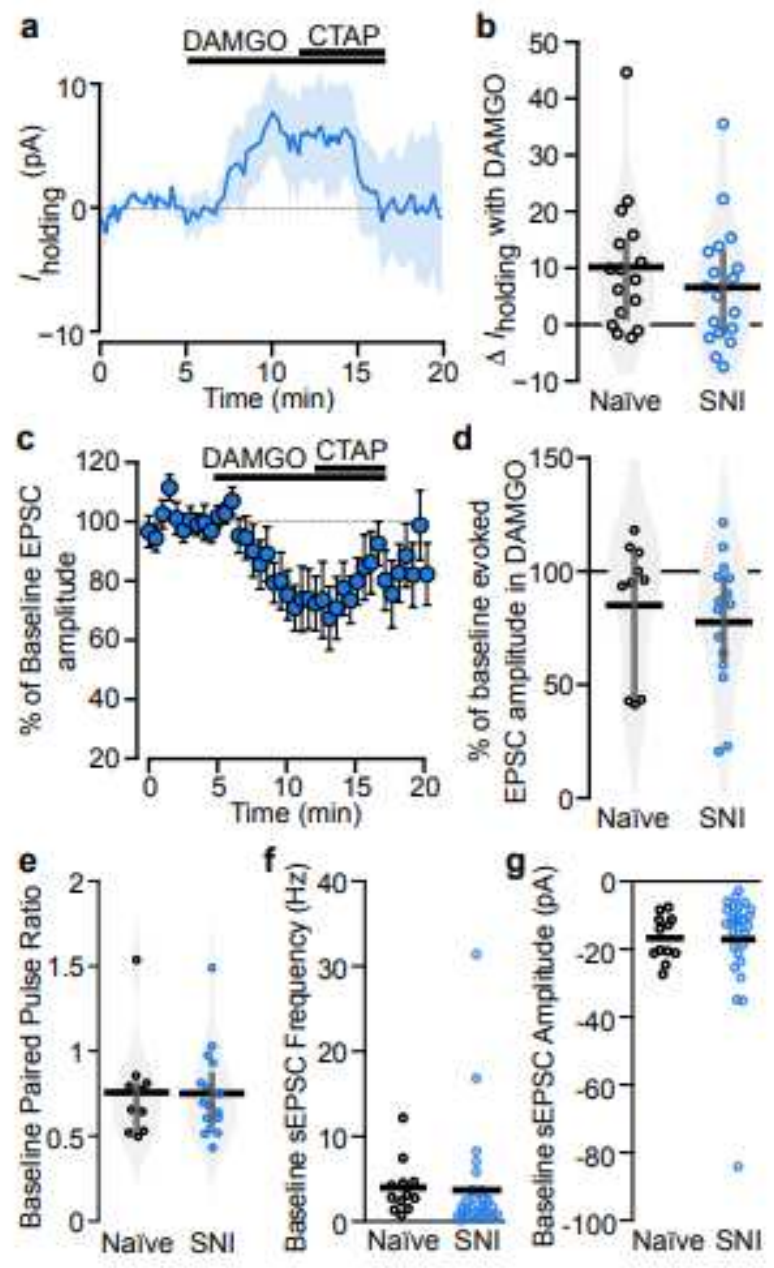

\section{Figure 2}

Ongoing pain does not alter MOR agonist synaptic effects or probability of glutamate release in the LHb. Responses to the MOR agonist DAMGO were recorded in voltage clamp, Vm $=-60 \mathrm{mV}$. a, In animals with $\mathrm{SNI}$, a subset of neurons responded to bath application of the MOR agonist DAMGO (500 nM) with a time locked outward current that was reversed with the MOR selective antagonist CTAP ( $500 \mathrm{nM})$. b, Postsynaptic holding current changes in response to bath application of DAMGO were not different between neurons from naïve and SNI rats: Unpaired t-test $d f=32, t=0.892, p=0.379$. $c$, In rats with SNI, DAMGO (500 nM) induced a time locked inhibition of electrically evoked glutamatergic EPSCs, and CTAP $(500 \mathrm{nM})$ reversed the effect. $d$, This DAMGO inhibition of electrically evoked EPSC amplitude was not different between LHb neurons from naïve and SNI rats: Unpaired t-test $d f=21, t=0.137, p=0.89$.

Baseline probability of release at glutamatergic synapses was similar between $\mathrm{LHb}$ neurons in naïve and SNI rats as measured by (e) paired pulse ratio (P2/P1) of electrically stimulated EPSCs (50 ms inter stimulus interval): Unpaired t-test, $d f=24, t=0.102, p=0.92$; $f$ ) baseline spontaneous EPSC frequency: Unpaired t-test unequal variances, $\mathrm{df}=37, \mathrm{t}=-0.17, \mathrm{p}=0.87$; and $(\mathrm{g})$ spontaneous EPSC amplitude: Unpaired t-test unequal variances, $\mathrm{df}=38, \mathrm{t}=-0.09, \mathrm{p}=0.93$. Data from naïve rats previously published in16. 


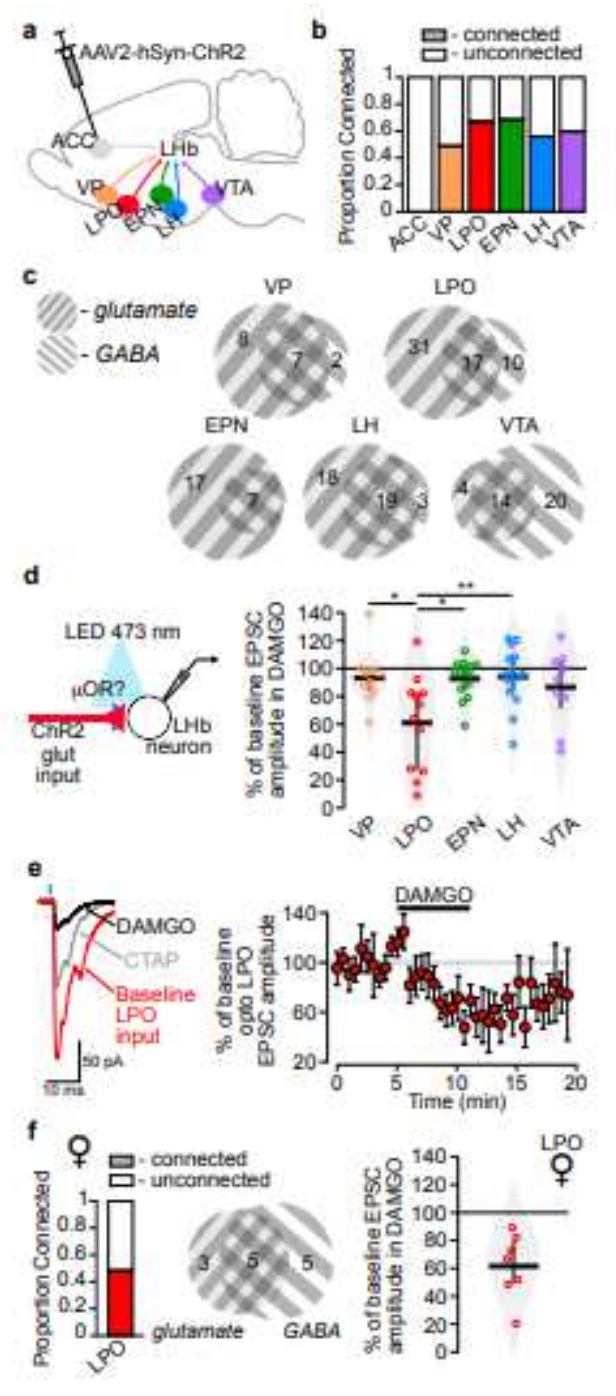

\section{Figure 3}

Glutamatergic inputs to the LHb from the LPO are inhibited by MOR activation. a, Schematic diagram of AAV2-hSynhChR2(H134R)-mCherry injection targets which project to the LHb. b, Proportions of LHb neurons receiving functional synaptic connections from six input regions measured with optogenetic stimulation of terminals expressing ChR2. c, Among connected neurons, venn diagrams depict the numbers of LHb cells receiving optically stimulated glutamatergic and/or GABAergic synaptic input. d, (Left) Experiment design: DAMGO (500 nM) was applied during optical synaptic stimulation experiments to probe for functional MOR presynaptic control of glutamate release from specific inputs. (Right) Change from baseline amplitude of optically-evoked EPSCs during DAMGO application. EPSCs from LPO terminal stimulation were most strongly inhibited by MOR activation: One-way ANOVA, $d f=4, F=4.11, p$ $=0.0057$ followed by Tukey HSD pairwise comparisons. e, (Left) Representative traces of opticallyevoked EPSCs in an LHb neuron from a rat with ChR2 expressed in inputs from the LPO. DAMGO decreased the amplitude of light-evoked EPSC, which was partially reversed by the selective MOR antagonist CTAP (500 nM). Rise time was $<2 \mathrm{~ms}$, indicative of a monosynaptic response. (Right) Summary time course of the DAMGO inhibition across all optically-evoked LPOLHb EPSCs $(n=12) . f$, LPO inputs to the LHb in female rats (left) had a similar overall connectivity rate but (middle) more 
GABAergic connections than glutamate connections. (Right) DAMGO inhibited light-evoked glutamatergic synaptic inputs from the LPO to the LHb in female rats as well.

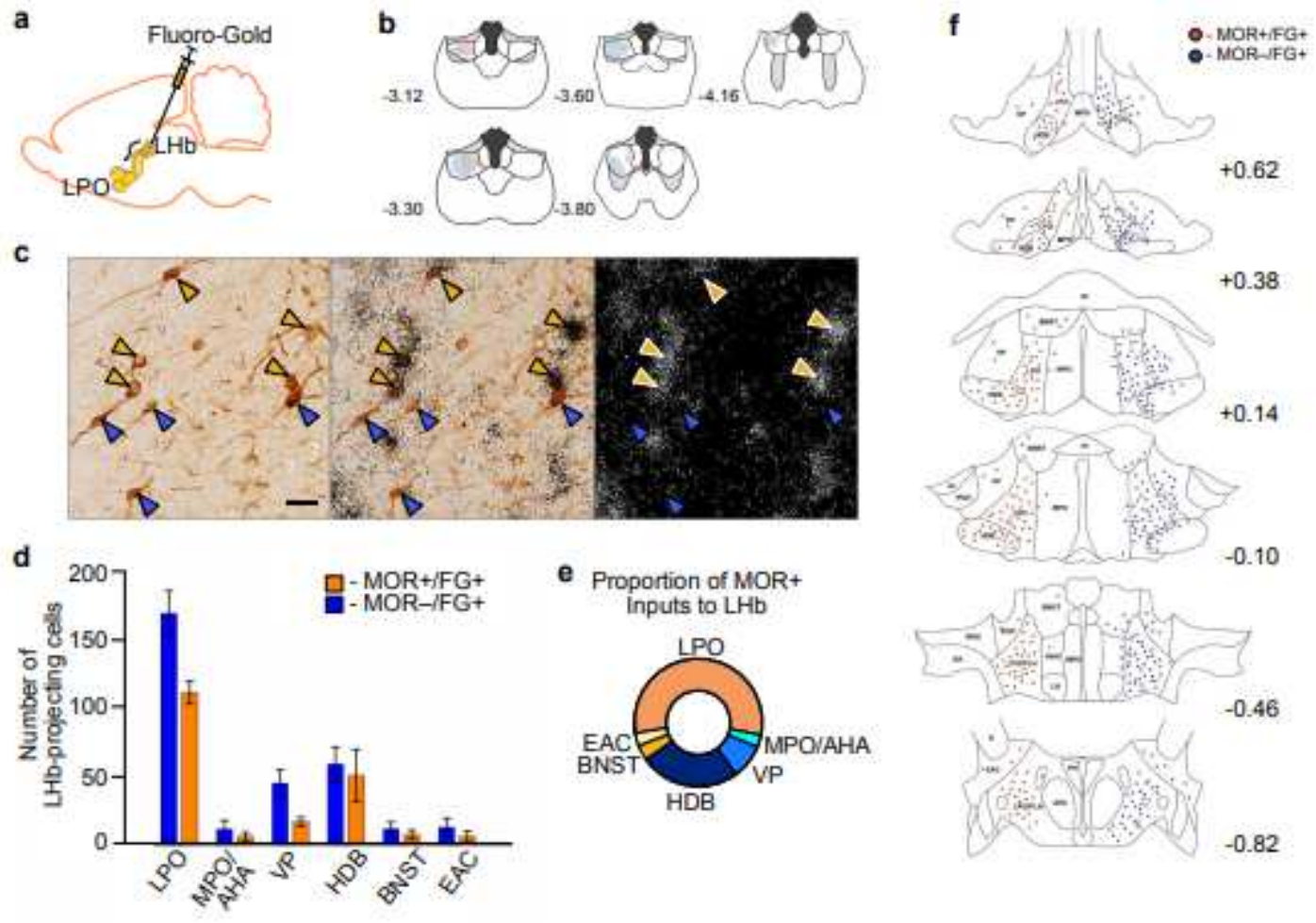

\section{Figure 4}

LPO inputs to the LHb highly express MOR mRNA. a, Schematic of unilateral iontophoretic deposition of the retrograde tracer FG into the LHb. b, FG injection sites in LHb ( $n=3$ rats). c, (Left and middle) Representative brightfield images of FG-positive/MOR-positive somas (yellow arrowhead) and FGpositive/MOR-negative somas (blue arrowhead) in the LPO. Scale bar $=20 \mu \mathrm{m}$. (Right) Darkfield image of in situ hybridization localization for MOR mRNA (OPRM1). d, Number of MOR-positive/FG-positive (yellow) and MOR-negative/FG-positive (blue) cells in various basal forebrain regions. e, Donut plot depicting proportions of total MOR-positive inputs to the LHb from various basal forebrain regions. $f$, Locations of FG-positive/MOR-positive (red) and FG-positive/MOR-negative (blue) somata throughout basal forebrain. Co-labeled cells are separated from the singly labelled population for display purposes. 


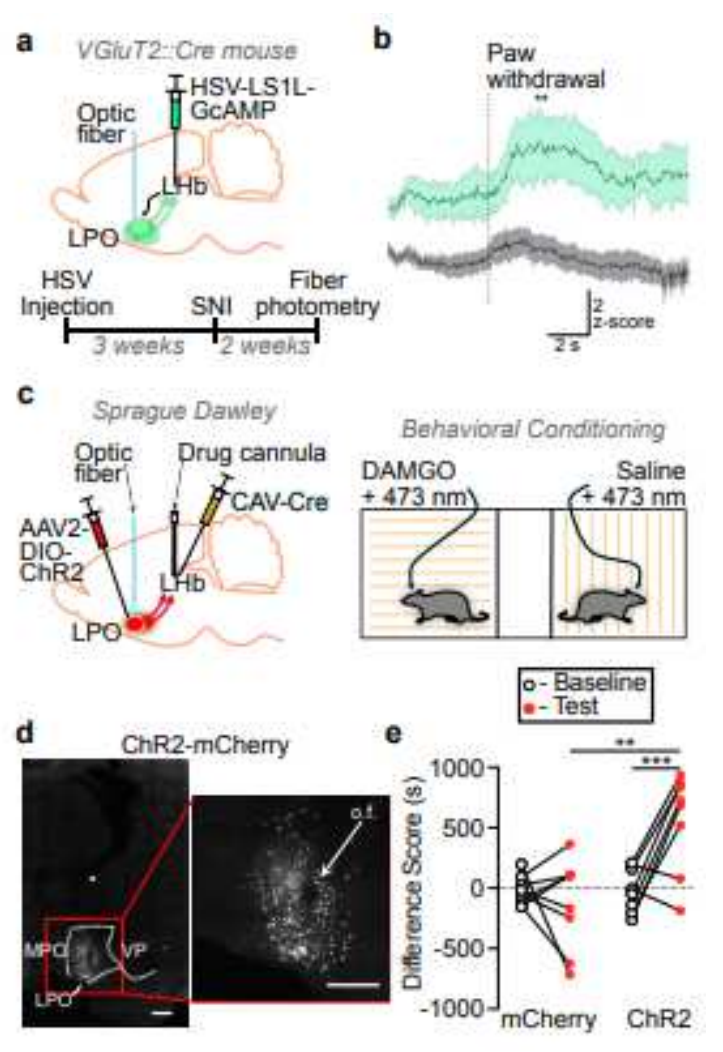

Figure 5

LHb MOR activation relieves aversiveness of glutamatergic LPO input to the LHb. a, (Top) Schematic diagram of HSV-hEF1aLS1L-GCaMP6m injection into the LHb of VGluT2::Cre mice, to induce expression of GCaMP6m in glutamatergic LPO neurons innervating the LHb, and optic fiber implant for fiber photometry imaging in the LHb-projecting LPO neurons. (Bottom) Experimental timeline. b, Mice with SNI $(n=5)$ exhibited significantly larger changes in GCaMP6m fluorescence time-locked to paw withdrawal in response to thermal stimulation in the Hargreaves apparatus compared to sham controls $(n=9)$ : Twoway ANOVA, $F(1,12)=5.439, p=0.038$; Holm-Sidak post-hoc test, $p=0.0074$. $c$, (Left) Schematic diagram of dual virus injections to express ChR2-mCherry or mCherry selectively in LPO neurons that project to the $\mathrm{LHb}$, optic fiber implantation targeting the LPO cell bodies, and cannula implantation targeting the LHb for experiments in male rats. (Right) Rats received bilateral blue light stimulation of the LPO during all conditioning sessions and in both chambers, sides paired with intraLHb saline or DAMGO. d, Representative images of Cre-dependent ChR2-mCherry fluorescence in LPO cell bodies. (Left) Scale bar = $250 \mu \mathrm{m}$. (Right) Lesion from optic fiber ("o.f.") implant amidst ChR2-mCherry-expressing cell bodies. Scale bar $=50 \mu$ m. e, Only animals with active ChR2 $(n=8)$, but not mCherry controls $(n=8)$, developed a preference for the intra-LHb DAMGO paired side of the place conditioning apparatus. Two-way mixed ANOVA, significant interaction between ChR2-mCherry/mCherry and baseline/test $F(1,14)=9.982, p=$ 0.007; post hoc effect group on test day adjusted $p=0.006$; paired t-tests, mCherry adjusted $p=0.427$; ChR2-mCherry adjusted $p=0.0027 .{ }^{* \star} p<0.01,{ }^{\star \star \star} p<0.005$ 